\title{
Stellar substructures in the solar neighbourhood ${ }^{\star}$
}

\section{Kinematic Group 1 in the Geneva-Copenhagen survey}

\author{
R. Ženoviené ${ }^{1}$, G. Tautvaišiené ${ }^{1}$, B. Nordström ${ }^{2,3}$, E. Stonkuté ${ }^{1}$, and G. Barisevičius ${ }^{1}$ \\ 1 Institute of Theoretical Physics and Astronomy, Vilnius University, A. Gostauto 12, 01108 Vilnius, Lithuania \\ e-mail: [renata.zenoviene;grazina.tautvaisiene; edita.stonkute; gintaras.barisevicius]@tfai.vu.lt \\ 2 Dark Cosmology Centre, Niels Bohr Institute, Copenhagen University, Juliane Maries Vej 30, 2100 Copenhagen, Denmark \\ 3 Stellar Astrophysics Centre, Department of Physics and Astronomy, Aarhus University, Ny Munkegade 120, 8000 Aarhus C, \\ Denmark \\ e-mail: birgitta@nbi.ku.dk
}

Received 30 September 2014 / Accepted 12 January 2015

\begin{abstract}
Context. A combined study of kinematics and chemical composition of stars is one of the most promising tools of research in Galaxy formation. The main goal in this field of research is to reconstruct the formation history of our Galaxy, to reveal the origin of the thick disc, and to find remnants of ancient mergers.

Aims. We determine detailed elemental abundances in stars belonging to the so-called Group 1 of the Geneva-Copenhagen survey (GCS) and compare the chemical composition with the Galactic thin- and thick-disc stars, with the GCS Group 2 and Group 3 stars, as well as with several kinematic streams of similar metallicities. The aim is to search for chemical signatures that might give information about the formation history of this kinematic group of stars.

Methods. High-resolution spectra were obtained with the Fibre-fed Echelle Spectrograph spectrograph at the Nordic Optical Telescope, La Palma, and were analysed with a differential model atmosphere method. Comparison stars were observed and analysed with the same method.

Results. The average value of $[\mathrm{Fe} / \mathrm{H}]$ for the 37 stars of Group 1 is $-0.20 \pm 0.14$ dex. Investigated Group 1 stars can be separated into three age subgroups. Along with the main 8- and 12-Gyr-old populations, a subgroup of stars younger than 5 Gyr can be separated as well. Abundances of oxygen, $\alpha$-elements, and r-process dominated elements are higher than in Galactic thin-disc dwarfs. This elemental abundance pattern has similar characteristics to that of the Galactic thick disc and differs slightly from those in Hercules, Arcturus, and AF06 stellar streams.

Conclusions. The similar chemical composition of stars in Group 1, as well as in Group 2 and 3, with that in stars of the thick disc might suggest that their formation histories are linked. The chemical composition pattern together with the kinematic properties and ages of stars in the investigated GCS groups provide evidence of their common origin and possible relation to an ancient merging event. A gas-rich satellite merger scenario is proposed as the most likely origin.
\end{abstract}

Key words. stars: abundances - Galaxy: disk - Galaxy: formation - Galaxy: evolution

\section{Introduction}

In this series of papers (Stonkutè et al. 2012, 2013, hereafter Papers I and II; Ženovienè et al. 2014, hereafter Paper III), we investigate the detailed chemical composition of stellar kinematic groups that were identified in the Geneva-Copenhagen survey (GCS, Nordström et al. 2004) and were suggested to belong to remnants of ancient merger events in our Galaxy (Helmi et al. 2006).

The formation and evolution of the Milky Way galaxy is one of the greatest outstanding questions of astrophysics. According Lambda cold dark matter $(\Lambda \mathrm{CDM})$ cosmological model, large galaxies like our Galaxy emerge as an end-point of hierarchical clustering, merging, and accretion, but we still lack the detailed physical picture of how individual stellar populations can be associated with elements of the proto-cloud, and how different Galactic components formed and evolved. Even the thick disc,

* Tables 4 and 5 are available in electronic form at http://www. aanda.org as a unique Galactic component discovered more than 30 years ago (Gilmore \& Reid 1983), still has no approved formation scenario. Separation of the Galactic disc into "thick" and "thin" disc populations refers to different types of stars. Thick-disc stars are essentially older and more highly enriched in $\alpha$-elements than thin-disc stars (e.g. Soubiran \& Girard 2005). The enhancement in $\alpha$-elements suggests that the thick-disc stars were formed on relatively short timescales ( $\sim 1 \mathrm{Gyr})$, thus offering us a hint to the formation history of our Galaxy (Kordopatis et al. 2013a). Ancient minor mergers of satellite galaxies are considered as one possible scenario of the thick-disc formation.

Numerical simulations of merger events have shown that such debris streams survive as coherent structures over gigayears (Helmi 2004; Law et al. 2005; Peñarrubia et al. 2005). Stellar streams may be discovered as overdensities in the phase space distribution of stars in the solar vicinity. Examining the GCS catalogue, Helmi et al. (2006) looked for stellar streams in a space of orbital apocentre, pericentre, and $z$-angular momentum $\left(L_{z}\right)$, the so-called APL-space. In this kind of space stellar streams 

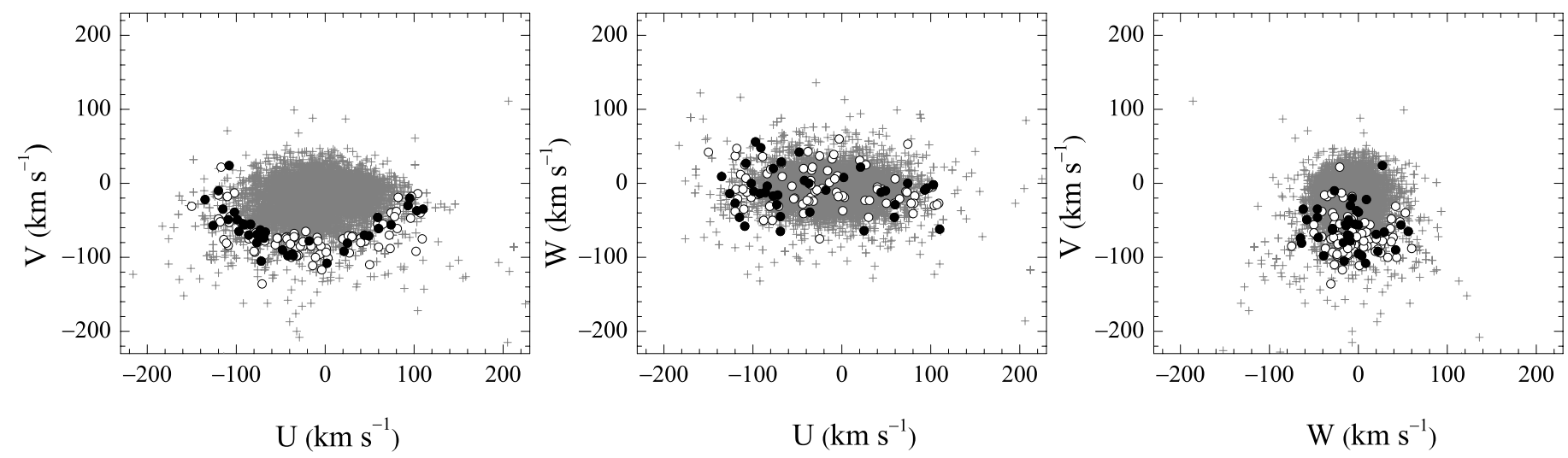

Fig. 1. Velocity distribution for all stars in the sample of Holmberg et al. (2009; plus signs), stars of Group 1 (circles), and the stars investigated here (filled circles).

cluster around lines of constant eccentricity. They found three new coherent stellar groups (Group 1, 2, and 3) with distinctive ages, metallicities, and kinematics, and suggested that those groups might correspond to remains of disrupted satellites.

Arifyanto \& Fuchs (2006), Dettbarn et al. (2007), and Klement et al. (2008) proposed an alternative projection of the eccentricity and $L_{\mathrm{z}}$ space, which according to Dekker's (1976) theory of Galactic orbits can be approximated by $\left(U^{2}+2 V^{2}\right)^{1 / 2}$ and $V$ velocities, respectively ( $U$ is pointing towards the Galactic center, $V$ into the direction of Galactic rotation). The assumption is that stars in the same stellar stream move on orbits that stay close together, which is justified by numerical simulations of satellite disruptions (Helmi et al. 2006). With this method, Arifyanto \& Fuchs (2006) identified several known streams (Hyades-Pleiades, Hercules, and Arcturus) and one new, the socalled AF06 stream (later confirmed by Klement et al. 2009 and by 2011).

Dettbarn et al. (2007) analysed the phase space distribution in a sample of non-kinematically selected low metallicity stars in the solar vicinity and determined the orbital parameters of several halo streams. One of those streams seemed to have precisely the same kinematics as the Sagittarius stream.

Klement et al. (2008) searched for stellar streams or moving groups in the solar neighbourhood, using data provided by the first RAVE public data release. They estimated overdensities related to the Sirius, Hercules, Arcturus, and HyadesPleiades moving groups. Besides, they found a new stream candidate (KFR08), suggesting that its origin is external to the Milky Way's disc. The KFR08 stream later was confirmed by Klement et al. (2009) and Bobylev et al. (2010). Antoja et al. (2012) also used the RAVE data and discovered a new group at $(U, V)=(92,-22) \mathrm{km} \mathrm{s}^{-1}$ in the solar neighbourhood. The new group was detected as a significant overdensity in the velocity distributions using a technique based on the wavelet transform.

A mechanism, known as "ringing" was introduced by Minchev et al. (2009). Minchev et al. (2009) showed that the sudden energy kick imparted by the gravitational potential of the satellite as it crosses the plane of the disc can strongly perturb the velocity field of disc stars located in local volumes such as the solar neighbourhood. These perturbations can be observed in the $(U, V)$ plane as arc-like features travelling in the direction of positive $V$ (see Gómez et al. 2012). Using this technique Minchev et al. (2009) confirmed presence of several known moving groups and predicted four new features at $V \sim-140,-120$, $40,60 \mathrm{~km} \mathrm{~s}^{-1}$. By matching the number and positions of the observed streams, they estimated that the Milky Way disc was strongly perturbed about 1.9 Gyr ago.

Various studies have been searching for evidence of minormerger events in the Milky Way (see review by Klement 2010). Large and deep chemodynamical surveys such as Gaia (Perryman et al. 2001) will enable us to characterise the Galaxy more precisely and to examine its formation scenarios as well as the kinematic stellar group identification methods.

An indispensable method to check the origin of various kinematic streams is the investigation of their chemical composition. In this paper, we present the detailed chemical composition of stars belonging to Group 1 of the Geneva-Copenhagen survey, which was identified by Helmi et al. (2006).

High-resolution spectra of 21 stars in the most metaldeficient GCS Group 3 were investigated in Papers I and II. Paper III presented results of the analysis of 32 stars in the GCS Group 2. Galactic thin-disc stars were analysed for a comparison as well. We present the detailed chemical composition results for Group 1, the last group of GCS. This kinematic group, which is the most numerous and metal-rich among the GCS groups, consists of 120 stars. According to the (Nordström et al. 2004) catalogue, its mean photometric metallicity, $[\mathrm{Fe} / \mathrm{H}]$, is about -0.45 dex. The Group 1 stars are distributed into two age populations of $8 \mathrm{Gyr}$ and 12 Gyr. Group 1 also differs from the other two groups by different metallicity and kinematics, and tends to have a significantly smaller $z$-velocity dispersion. We investigated chemical composition for 37 Group 1 stars, eight comparison Galactic thin-disc stars, and five comparison Galactic thick-disc stars. We determined abundances of up to 22 chemical elements and compared them with the Galactic disc abundance pattern.

In Fig. 1, we show the velocity distribution of the Galactic disc stars from Holmberg et al. (2009). Stars belonging to GCS Group 1 are marked with open and filled circles (the latter are used to mark stars we investigated). An average error of stellar space motions in each component $\left(U, V\right.$, and $W$ ) is $1.5 \mathrm{~km} \mathrm{~s}^{-1}$ (Nordström et al. 2004). It is clear from Fig. 1 that the distribution of Group 1 stars in the velocity space differs in comparison to other stars of the Galactic disc. For example in the $(U, V)$ plane Group 1 stars are distributed in a banana shape, whereas the thindisc stars define a centrally concentrated clump. These characteristic patterns are the same as those of infalling dwarf satellites after several gigayears (see Helmi et al. 2006 for a comprehensive discussion). In Fig. 2, the stars are shown in the APL-space. Kinematic group stars spread out in the edge of the distribution of data points. The location of a star in the APL space is very 
R. Ženovienè et al.: Stellar substructures in the solar neighbourhood. IV.

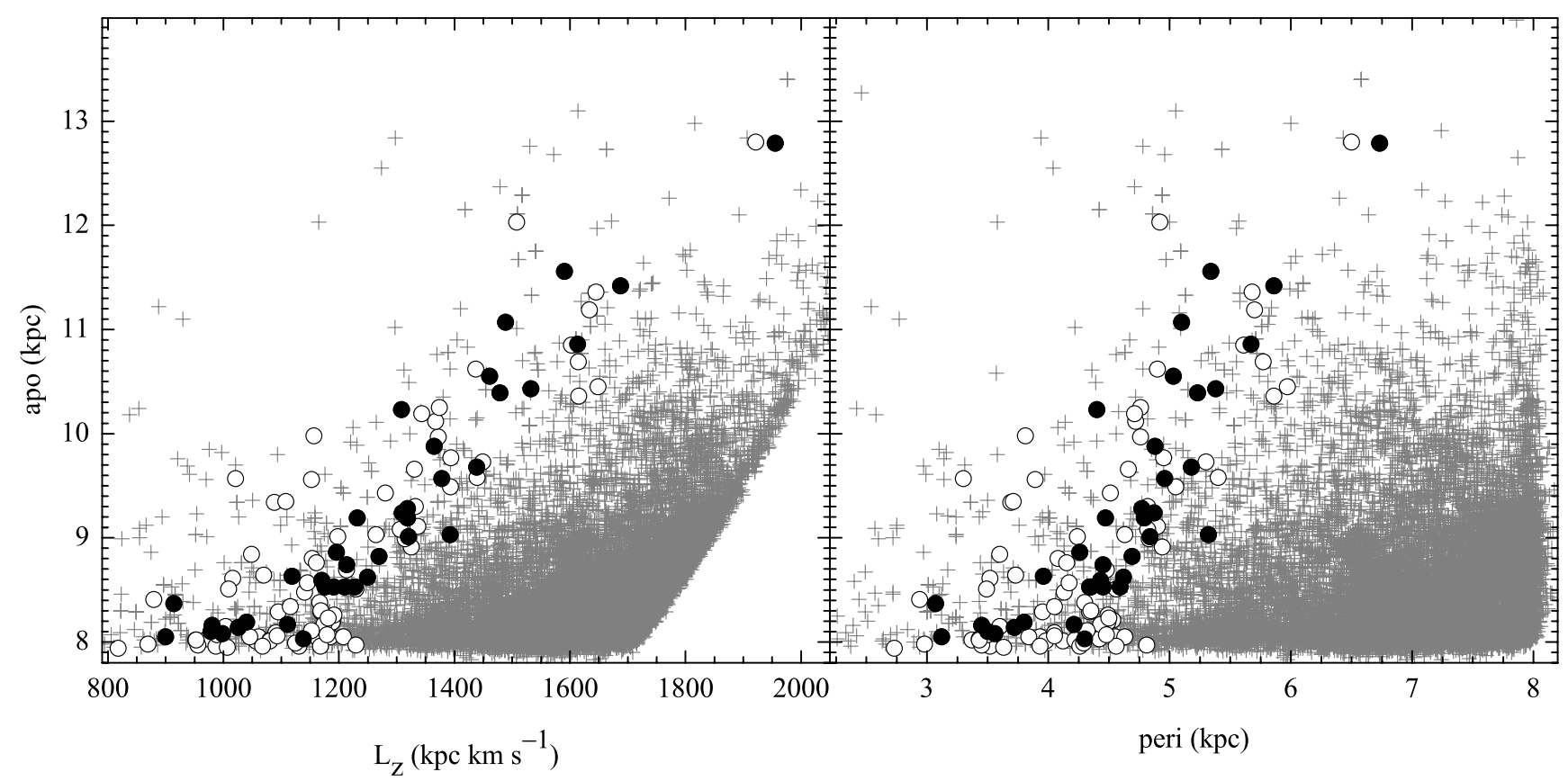

Fig. 2. Distribution for the stars in the APL space. Plus signs denote the GCS sample (Holmberg et al. 2009), circles denote Group 1, the filled circles are the stars we investigated. Note that these stars as well as all Group 1 stars are distributed in APL space with constant eccentricity.

accurate. A limited knowledge of the form of the Galactic potential, used to determine values of apocentre and pericentre, does not affect the distribution of points in the APL space much because the volume probed by the GCS sample is so small that the Galactic potential is close to a constant inside this region (cf. Helmi et al. 2006). This implies that the energy, and hence the orbital parameters or the location of a star in the APL space, are determined mostly by its kinematics rather than by its spatial location (or the Galactic potential). Changes in the Galactic potential produce only small variations in the orbital parameters. According to Helmi et al. (2006), in the case of the potential proposed by Flynn et al. (1996), which was used for the orbit integrations in Nordström et al. (2004) and the following papers, a typical change in the apocentre and pericentre is of about 1-2\%.

Despite the fact that the kinematic group members, over time, were dispersed through the Galactic disc, their chemical composition should remain unchanged. The high-resolution spectroscopic analysis is an important supplemental method in revealing and confirming the origin and history of the GCS stellar groups.

\section{Observations and analysis}

We obtained high-resolution spectra of the programme and comparison stars with the Fibre-fed Echelle Spectrograph (FIES) on the Nordic Optical $2.5 \mathrm{~m}$ telescope (NOT) during 2008, 2011, and 2012. This spectrograph gives spectra of resolving power $(R \approx 68000)$ in the wavelength range of 3680-7270 $\mathrm{A}$. We exposed the spectra to reach a signal-to-noise ratio higher than 100 . We elected the programme stars so that they were observable from the NOT and were brighter than $V=10 \mathrm{mag}$. Reductions of CCD images were made with the FIES pipeline FIES tool, which performs a complete reduction: calculation of reference frame, bias and scattering subtraction, flat-field division, wavelength calibration and other procedures ${ }^{1}$. A list of the observed
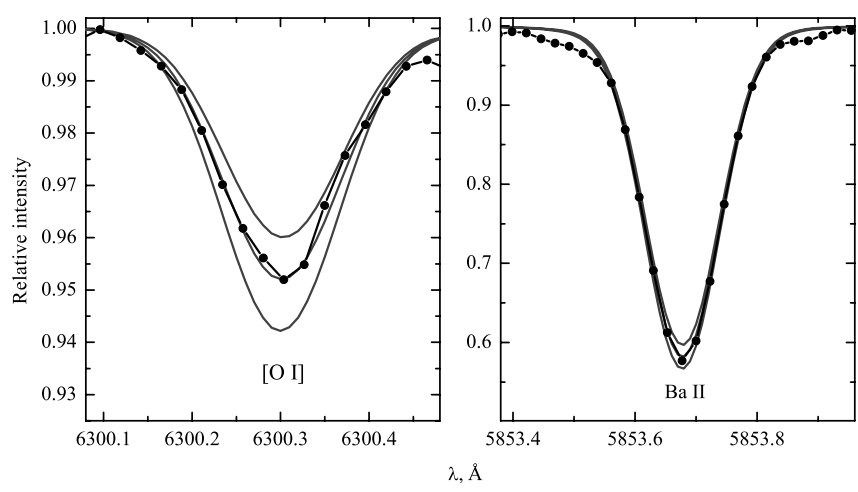

Fig. 3. Synthetic spectrum fit to the forbidden [O I] line at $6300.3 \AA$ in the observed spectrum of HD 157214 (left panel) and to the barium line at $5853 \AA$ in the observed spectrum of HD 52711. The observed spectra are shown by solid lines with dots. The dark grey solid lines are synthetic spectra with $[\mathrm{O} / \mathrm{Fe}]=0.42 \pm 0.1$ and with $[\mathrm{Ba} / \mathrm{Fe}]=$ $-0.08 \pm 0.1$, respectively.

stars and some of their parameters (taken from the catalogue of Holmberg et al. 2009 and SIMBAD) are presented in Table 1.

We analysed the spectra using the differential model atmosphere technique described in Papers I-III. Here we will revisit only a few details. The Eqwidth and BSYN program packages, developed at the Uppsala Astronomical Observatory, were used to carry out the calculation of abundances from measured equivalent widths and synthetic spectra, respectively. A set of planeparallel, line-blanketed, constant-flux LTE model atmospheres (Gustafsson et al. 2008) were taken from the MARCS stellar model atmosphere and flux library ${ }^{2}$.

We used the Vienna Atomic Line Data Base (VALD, Piskunov et al. 1995) to prepare input data for the calculations. We took the atomic oscillator strengths for the main spectral 
Table 1. Parameters of programme and comparison stars.

\begin{tabular}{|c|c|c|c|c|c|c|c|c|c|c|c|c|}
\hline Star & Sp. type & $\begin{array}{l}\text { Age } \\
\text { H09* }\end{array}$ & $\begin{array}{l}\text { Age } \\
\text { C11* }\end{array}$ & $\begin{array}{c}M_{V} \\
\text { mag }\end{array}$ & $\begin{array}{c}d \\
\mathrm{pc}\end{array}$ & $\begin{array}{c}U \\
\mathrm{~km} \mathrm{~s}^{-1} \\
\end{array}$ & $\begin{array}{c}V \\
\mathrm{~km} \mathrm{~s}^{-1} \\
\end{array}$ & $\begin{array}{c}W \\
\mathrm{~km} \mathrm{~s}^{-1} \\
\end{array}$ & $e$ & $\begin{array}{l}z_{\max } \\
\mathrm{kpc}\end{array}$ & $\begin{array}{l}R_{\text {peri }} \\
\mathrm{kpc}\end{array}$ & $\begin{array}{l}R_{\text {apo }} \\
\mathrm{kpc}\end{array}$ \\
\hline \multicolumn{13}{|c|}{ Group 1 stars } \\
\hline HD 3795 & K0V & 10.6 & 9.9 & 3.84 & 29 & -48 & -90 & 42 & 0.37 & 1.07 & 3.80 & 8.19 \\
\hline HD 4607 & F5 & 4.2 & 3.5 & 3.08 & 85 & -108 & 24 & 27 & 0.31 & 0.86 & 6.73 & 12.79 \\
\hline HD 15777 & G0 & 11 & 11.1 & 4.28 & 50 & -77 & -69 & 20 & 0.32 & 0.50 & 4.45 & 8.74 \\
\hline HD 22872 & F9V & 6.2 & 5.9 & 3.59 & 74 & -99 & -49 & -11 & 0.32 & 0.06 & 4.96 & 9.57 \\
\hline HD 25123 & G0 & 11 & $\ldots$ & 4.01 & 67 & 93 & -30 & -9 & 0.32 & 0.03 & 5.38 & 10.43 \\
\hline HD 40040 & G0 & 12.4 & 11 & 4.12 & 65 & 44 & -70 & -12 & 0.31 & 0.07 & 4.45 & 8.53 \\
\hline HD 49409 & G0V & 11.2 & 8.2 & 4.30 & 53 & 110 & -35 & -62 & 0.37 & 1.53 & 5.10 & 11.07 \\
\hline HD 52711 & G4V & 7.9 & 5.1 & 4.53 & 19 & -18 & -78 & -9 & 0.30 & 0.03 & 4.30 & 8.03 \\
\hline HD 60779 & G0 & 6.8 & 5.3 & 4.38 & 36 & -126 & -57 & -14 & 0.40 & 0.11 & 4.40 & 10.23 \\
\hline HD 67088 & G5 & 13.7 & $\ldots$ & 4.63 & 67 & 95 & -20 & -7 & 0.31 & 0.05 & 5.67 & 10.86 \\
\hline HD 67587 & F8 & 4.7 & 3.9 & 3.29 & 47 & 74 & -56 & 0 & 0.32 & 0.12 & 4.77 & 9.28 \\
\hline HD 76095 & G5V & 5.5 & 5.3 & 3.28 & 49 & 2 & -108 & 8 & 0.44 & 0.25 & 3.12 & 8.05 \\
\hline HD 77408 & F6IV & 3.7 & 4.3 & 3.52 & 50 & -120 & -10 & -27 & 0.32 & 0.40 & 5.86 & 11.42 \\
\hline HD 78558 & G0 & 11.6 & 10.9 & 4.44 & 37 & -69 & -74 & -65 & 0.32 & 1.24 & 4.43 & 8.59 \\
\hline HD 88371 & G2V & 13.2 & 9.4 & 4.55 & 59 & -135 & -22 & 9 & 0.37 & 0.31 & 5.34 & 11.56 \\
\hline HD 88446 & F8 & 7.3 & 7.8 & 3.77 & 67 & -42 & -98 & 4 & 0.41 & 0.17 & 3.45 & 8.16 \\
\hline HD 90508 & G1V & 10.4 & 7.4 & 4.63 & 23 & 21 & -92 & 22 & 0.37 & 0.51 & 3.72 & 8.14 \\
\hline HD 109498 & G3V & 10.4 & $\ldots$ & 4.53 & 69 & 59 & -46 & -46 & 0.26 & 0.79 & 5.32 & 9.03 \\
\hline HD 111367 & G1V & 9.5 & 8.5 & 4.11 & 86 & -69 & -73 & -45 & 0.33 & 0.76 & 4.34 & 8.53 \\
\hline HD 135694 & K0 & 12.8 & 6.7 & 4.82 & 71 & -36 & -98 & -39 & 0.40 & 0.57 & 3.50 & 8.10 \\
\hline HD 138750 & F8 & 3.3 & 3.2 & 2.60 & 116 & -72 & -105 & -16 & 0.46 & 0.18 & 3.07 & 8.37 \\
\hline HD 140209 & G0 & 10.4 & 9.6 & 4.03 & 71 & -73 & -63 & -29 & 0.30 & 0.37 & 4.62 & 8.62 \\
\hline HD 149105 & G0V & 6 & 5.8 & 3.37 & 53 & 49 & -71 & -10 & 0.32 & 0.06 & 4.35 & 8.53 \\
\hline HD 149890 & F8V & 6.8 & 6.1 & 4.15 & 39 & 60 & -61 & -29 & 0.31 & 0.38 & 4.69 & 8.82 \\
\hline HD 156617 & G5 & 9.3 & 9.1 & 3.92 & 66 & -102 & -39 & 0 & 0.30 & 0.11 & 5.18 & 9.68 \\
\hline HD 156893 & G5 & 8.9 & 8.5 & 3.74 & 77 & -97 & -65 & 56 & 0.35 & 1.60 & 4.47 & 9.19 \\
\hline HD 157214 & G0V & 13.9 & 8.1 & 4.60 & 14 & 25 & -81 & -64 & 0.32 & 1.33 & 4.21 & 8.17 \\
\hline $\mathrm{BD}+403374$ & K1 & & 6.4 & 6.43 & 49 & -109 & -49 & -58 & 0.34 & 1.15 & 4.88 & 9.88 \\
\hline HD 171009 & G5 & 11.7 & 6.7 & 4.24 & 66 & -68 & -66 & 29 & 0.30 & 0.68 & 4.59 & 8.53 \\
\hline HD 171242 & G0 & 9.2 & 8.5 & 4.02 & 62 & 103 & -37 & -2 & 0.35 & 0.10 & 5.03 & 10.55 \\
\hline HD 178478 & G5 & 15.8 & $\ldots$ & 4.90 & 47 & -86 & -70 & -12 & 0.35 & 0.08 & 4.26 & 8.86 \\
\hline HD 188326 & G8IV & $\ldots$ & 8.7 & 3.83 & 56 & -91 & -56 & 48 & 0.31 & 1.24 & 4.87 & 9.24 \\
\hline HD 206373 & G0V & 5.5 & 5.6 & 3.19 & 104 & -37 & -95 & 0 & 0.39 & 0.11 & 3.56 & 8.08 \\
\hline HD 210483 & G1V & 9.3 & 6.6 & 4.03 & 51 & -77 & -80 & -17 & 0.37 & 0.15 & 3.96 & 8.63 \\
\hline HD 211476 & $\mathrm{G} 2 \mathrm{~V}$ & 7.1 & 6.7 & 4.59 & 31 & -115 & -35 & -46 & 0.33 & 0.87 & 5.23 & 10.39 \\
\hline HD 217511 & F5 & 2 & 2.1 & 2.23 & 122 & -84 & -55 & -4 & 0.30 & 0.08 & 4.84 & 9.01 \\
\hline HD 219175 & F9V & 3.5 & 2.9 & 4.61 & 39 & -92 & -55 & -14 & 0.31 & 0.10 & 4.79 & 9.19 \\
\hline \multicolumn{13}{|c|}{ Thin-disc stars } \\
\hline HD 115383 & G0V & 4.3 & 5.1 & 3.97 & 18 & -38 & 2 & -18 & 0.09 & 0.18 & 7.54 & 8.99 \\
\hline HD 127334 & G5V & 10.5 & 10.7 & 4.51 & 24 & 30 & -4 & -2 & 0.12 & 0.08 & 7.14 & 9.10 \\
\hline HD 136064 & F9IV & 3 & 3.7 & 3.12 & 25 & 62 & -29 & -24 & 0.23 & 0.29 & 5.83 & 9.37 \\
\hline HD 163989 & F6IV & 2.2 & 2.5 & 2.51 & 32 & -29 & -27 & -22 & 0.10 & 0.22 & 6.64 & 8.16 \\
\hline HD 187013 & F7V & 2.8 & $\ldots$ & 3.34 & 22 & 38 & -8 & -25 & 0.14 & 0.28 & 6.88 & 9.20 \\
\hline HD 187691 & F8V & 3.3 & 3.1 & 3.71 & 19 & -3 & -3 & -25 & 0.03 & 0.27 & 7.88 & 8.28 \\
\hline HD 200790 & F8V & 2.2 & 2.6 & 2.51 & 49 & 19 & -37 & 10 & 0.15 & 0.27 & 6.03 & 8.21 \\
\hline HD 220117 & F5V & 1.8 & 1.6 & 2.66 & 42 & -15 & -21 & -16 & 0.07 & 0.12 & 7.03 & 8.02 \\
\hline \multicolumn{13}{|c|}{ Thick-disc stars } \\
\hline HD 150433 & G0 & 13.8 & 7.1 & 4.86 & 30 & -8 & -58 & -46 & 0.21 & 0.73 & 5.21 & 7.97 \\
\hline HD 181047 & G8V & 13.9 & 10.2 & 4.96 & 47 & -99 & -43 & -17 & 0.30 & 0.15 & 5.12 & 9.57 \\
\hline HD 186411 & G0 & 6 & $\ldots$ & 3.36 & 88 & -64 & -56 & -6 & 0.26 & 0.02 & 4.96 & 8.52 \\
\hline HD 195019 & $\mathrm{G} 3 \mathrm{IV}-\mathrm{V}$ & 9.7 & 8.6 & 3.96 & 39 & -73 & -76 & -39 & 0.34 & 0.61 & 4.18 & 8.57 \\
\hline HD 198300 & G0 & 10.3 & 7.1 & 4.89 & 53 & 81 & -20 & -24 & 0.28 & 0.28 & 5.84 & 10.26 \\
\hline
\end{tabular}

Notes. ${ }^{(*)}$ H 09 - ages taken from Holmberg et al. (2009); C11 - from Casagrande et al. (2011).

lines we analysed from an inverse solar spectrum analysis performed in Kiev (Gurtovenko \& Kostyk 1989).

Initial values of the effective temperatures for the programme stars were taken from Holmberg et al. (2009) and then carefully checked and corrected, if needed, by forcing Fe I lines to yield no dependency of iron abundance on excitation potential through changes to the model effective temperature. We used the ionisation equilibrium method to find surface gravities of the programme stars by forcing neutral and ionised iron lines to yield the same iron abundances. Microturbulence velocity values corresponding to the minimal line-to-line Fe I abundance scattering were chosen as correct values. We performed the spectral synthesis method for the determination of oxygen, yttrium, zirconium, barium, lanthanum, cerium, praseodymium, neodymium, samarium, and europium abundances. Several fits of the synthetic line profiles to the observed spectra are shown 
R. Ženovienè et al.: Stellar substructures in the solar neighbourhood. IV.

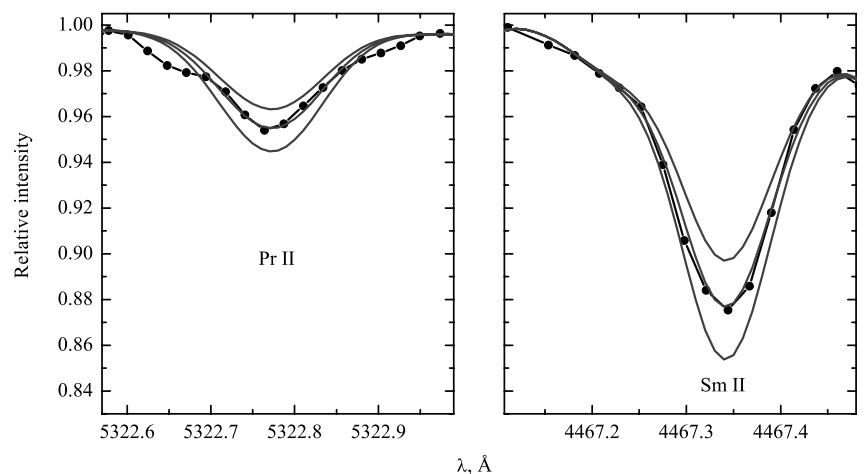

Fig. 4. Synthetic spectrum fit to the praseodymium line at $5323 \AA$ in the observed spectrum of HD 3795 (left panel) and to the samarium line at $4467 \AA$ in the observed spectrum of HD 22872. The observed spectra are shown by solid lines with dots. The dark grey solid lines are synthetic spectra with $[\mathrm{Pr} / \mathrm{Fe}]=0.54 \pm 0.1$ and $[\mathrm{Sm} / \mathrm{Fe}]=0.01 \pm 0.1$, respectively.

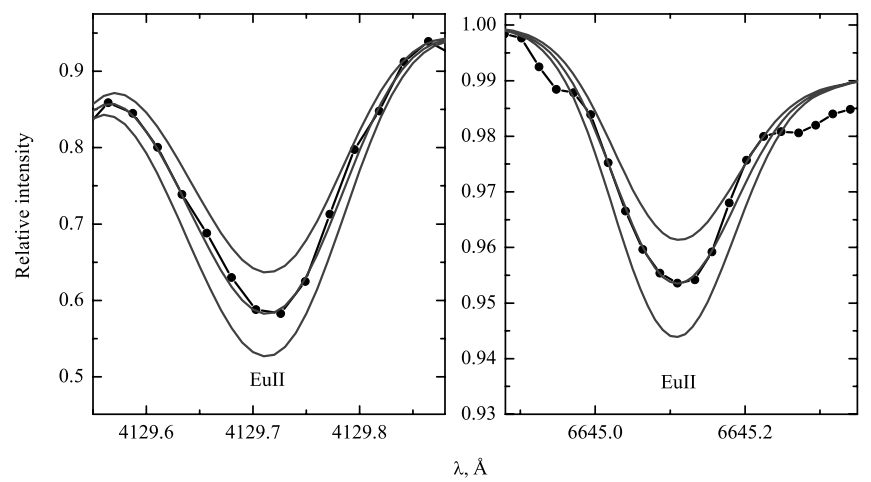

Fig. 5. Synthetic spectrum fit to the europium lines at $4129 \AA$ and $6645 \AA$. The observed spectrum for the programme star HD 149105 is shown as a solid line with dots. The dark grey solid lines are synthetic spectra with $[\mathrm{Eu} / \mathrm{Fe}]=0.04 \pm 0.1$ and $[\mathrm{Eu} / \mathrm{Fe}]=0.11 \pm 0.1$ for these two lines, respectively.

in Figs. 3-5. We complied atomic parameters of lines in the intervals of spectral syntheses from the VALD database. We calibrated all $\log g f$ values to fit to the solar spectrum by Kurucz (2005) with solar abundances from Grevesse \& Sauval (2000). We took hyperfine structures and isotope shifts into account as appropriate. Abundances of other chemical elements were determined using equivalent widths of their lines. We determined abundances of $\mathrm{Na}$ and $\mathrm{Mg}$ taking non-local thermodynamical equilibrium (NLTE) into account. The equivalent widths of the lines were measured by fitting of a Gaussian profile using the 4A software package (Ilyin 2000).

The uncertainties in abundances are due to several sources: uncertainties caused by analysis of individual lines, including random errors of atomic data and continuum placement and uncertainties in the stellar parameters. The sensitivity of the abundance estimates to changes in the atmospheric parameters by the assumed errors $\Delta[\mathrm{El} / \mathrm{H}]^{3}$ are illustrated for the star HD 52711 (Table 2). Clearly, possible parameter errors do not affect the abundances seriously; the element-to-iron ratios, which we use in our discussion, are even less sensitive.

The scatter of the deduced abundances from different spectral lines $\sigma$ gives an estimate of the uncertainty due to the

\footnotetext{
3 We use the customary spectroscopic notation $[\mathrm{X} / \mathrm{Y}] \equiv \log _{10}\left(N_{\mathrm{X}} / N_{\mathrm{Y}}\right)_{\mathrm{star}}-\log _{10}\left(N_{\mathrm{X}} / N_{\mathrm{Y}}\right)_{\odot}$.
}

Table 2. Effects on derived abundances resulting from model changes for the star HD 52711.

\begin{tabular}{lcccc}
\hline \hline Ion & $\Delta T_{\text {eff }}+100 \mathrm{~K}$ & $\Delta \log g+0.3$ & $\Delta v_{\mathrm{t}}+0.3 \mathrm{~km} \mathrm{~s}^{-1}$ & Total \\
\hline [O I & 0.03 & 0.13 & 0.01 & 0.13 \\
$\mathrm{Na}$ I & 0.05 & -0.02 & -0.01 & 0.05 \\
$\mathrm{Mg}$ I & 0.05 & -0.02 & -0.03 & 0.06 \\
$\mathrm{Al}$ I & 0.04 & 0.00 & 0.00 & 0.04 \\
$\mathrm{Si}$ I & 0.03 & 0.01 & -0.01 & 0.03 \\
$\mathrm{Ca}$ I & 0.07 & -0.01 & -0.02 & 0.07 \\
$\mathrm{Sc}$ II & -0.00 & 0.11 & -0.04 & 0.12 \\
Ti I & 0.09 & 0.00 & -0.01 & 0.09 \\
Ti II & 0.01 & 0.12 & -0.05 & 0.13 \\
V I & 0.11 & -0.00 & -0.01 & 0.11 \\
Cr I & 0.08 & -0.02 & -0.06 & 0.10 \\
Cr II & -0.03 & 0.10 & -0.09 & 0.14 \\
Fe I & 0.07 & -0.02 & -0.07 & 0.10 \\
Fe II & -0.02 & 0.11 & -0.07 & 0.13 \\
Co I & 0.08 & 0.01 & -0.01 & 0.08 \\
Ni I & 0.06 & -0.00 & -0.04 & 0.07 \\
Y II & 0.02 & 0.10 & -0.12 & 0.16 \\
Zr I & 0.11 & 0.01 & 0.01 & 0.11 \\
Zr II & 0.02 & 0.13 & 0.01 & 0.13 \\
Ba II & 0.06 & 0.08 & -0.18 & 0.21 \\
La II & 0.03 & 0.12 & 0.01 & 0.12 \\
Ce II & 0.03 & 0.11 & 0.01 & 0.11 \\
Pr II & 0.02 & 0.12 & 0.01 & 0.12 \\
Nd II & 0.03 & 0.12 & -0.01 & 0.12 \\
Sm II & 0.04 & 0.11 & -0.01 & 0.12 \\
Eu II & 0.03 & 0.12 & 0.01 & 0.12 \\
\hline
\end{tabular}

Notes. The table entries show the effects on the logarithmic abundances relative to hydrogen, $\Delta[\mathrm{El} / \mathrm{H}]$.

random errors. The mean value of $\sigma$ is 0.04 dex, thus the uncertainties in the derived abundances that are the result of random errors amount to approximately this value.

Effective temperatures for all stars investigated here are also available in Holmberg et al. (2009) and Casagrande et al. (2011). Casagrande et al. (2011) provided astrophysical parameters for the Geneva-Copenhagen survey by applying the infrared flux method to determine the effective temperature. In comparison to Holmberg et al. (2009), stars in the catalogue of Casagrande et al. (2011) are on average $100 \mathrm{~K}$ hotter. For the stars investigated here, our spectroscopic temperatures are on average only $40 \pm 70 \mathrm{~K}$ hotter than in Holmberg et al. (2009) and $40 \pm 90 \mathrm{~K}$ cooler than in Casagrande et al. (2011). The [Fe/H] values for all of the stars we investigated are available in Holmberg et al. (2009) as well as in Casagrande et al. (2011). A comparison between Holmberg et al. (2009) and Casagrande et al. (2011) shows that the latter gives $[\mathrm{Fe} / \mathrm{H}]$ values that are more metalrich on average by 0.1 dex. For our programme stars, we obtain a difference of $0.1 \pm 0.1$ dex in comparison with Holmberg et al. (2009) and we obtain no systematic difference, but a scatter of 0.1 dex, in comparison with Casagrande et al. (2011). The same result was found in comparing the atmospheric parameters determined for Group 3 stars in our Paper I and for Group 2 in Paper III.

Some stars from our sample were previously investigated by other authors. In Table 3, we present a comparison with results by Bensby et al. (2014) and Reddy et al. (2006), who investigated several stars in common with our work. Eight thin-disc stars that we investigated for a comparison have been analysed previously by Edvardsson et al. (1993). Slight differences in the $\log g$ values lie within the errors of uncertainties and are 

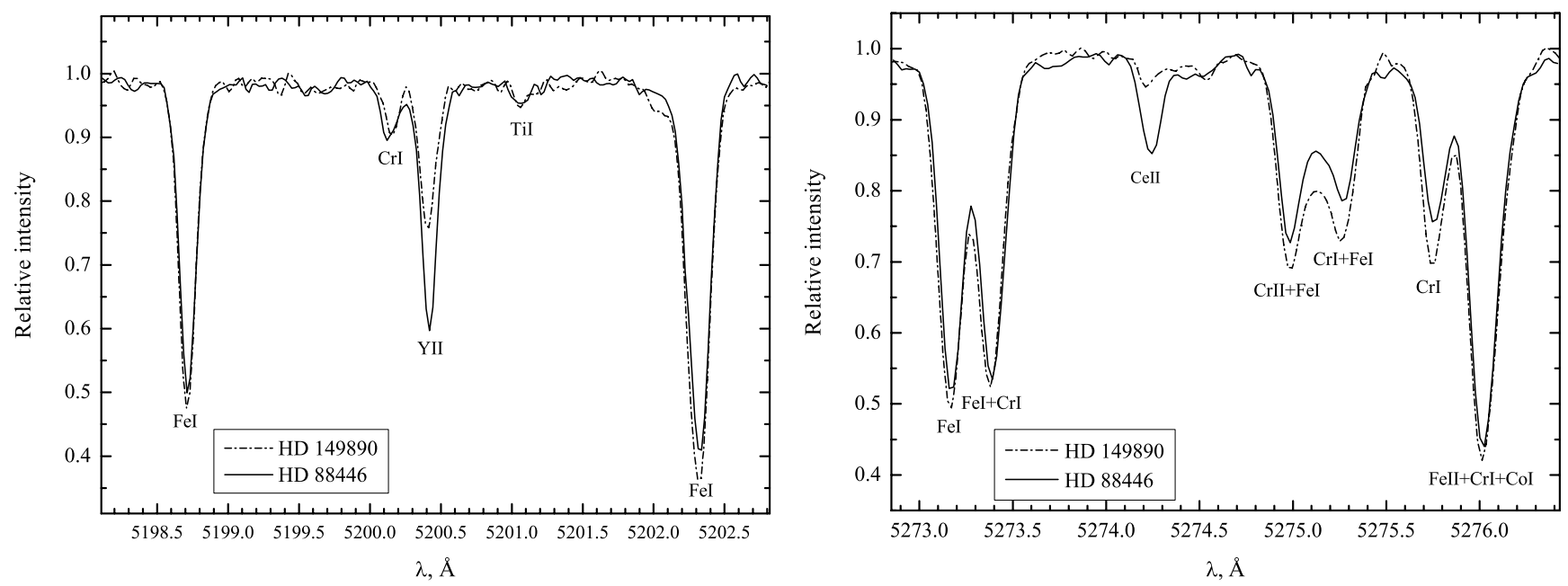

Fig. 6. NOT-FIES spectra of Group 1 stars HD 149890 and HD 88446. These two spectra are over-plotted to see the difference in spectral lines of elements produced in $s$-process, while lines of other chemical elements are similar.

Table 3. Comparison with previous studies.

\begin{tabular}{lccc}
\hline \hline Quantity & Ours-B14 & Ours-R06 & Ours-E93 \\
\hline$T_{\text {eff }}$ & $-31 \pm 48$ & $74 \pm 51$ & $-12 \pm 65$ \\
$\log g$ & $-0.18 \pm 0.10$ & $-0.16 \pm 0.15$ & $-0.28 \pm 0.24$ \\
{$[\mathrm{Fe} / \mathrm{H}]$} & $0.04 \pm 0.05$ & $0.09 \pm 0.06$ & $0.15 \pm 0.06$ \\
{$[\mathrm{Na} / \mathrm{Fe}]$} & $-0.01 \pm 0.03$ & $-0.05 \pm 0.05$ & $-0.05 \pm 0.07$ \\
{$[\mathrm{Mg} / \mathrm{Fe}]$} & $0.05 \pm 0.08$ & $-0.07 \pm 0.05$ & $0.06 \pm 0.11$ \\
{$[\mathrm{Al} / \mathrm{Fe}]$} & $0.00 \pm 0.06$ & $-0.02 \pm 0.06$ & $-0.11 \pm 0.05$ \\
{$[\mathrm{Si} / \mathrm{Fe}]$} & $0.01 \pm 0.03$ & $-0.03 \pm 0.03$ & $-0.02 \pm 0.03$ \\
{$[\mathrm{Ca} / \mathrm{Fe}]$} & $0.02 \pm 0.03$ & $0.04 \pm 0.02$ & $0.03 \pm 0.05$ \\
{$[\mathrm{Sc} / \mathrm{Fe}]$} & $\ldots$ & $0.01 \pm 0.07$ & $\ldots$ \\
{$[\mathrm{Ti} / \mathrm{Fe}]$} & $0.02 \pm 0.04$ & $0.06 \pm 0.04$ & $-0.04 \pm 0.06$ \\
{$[\mathrm{~V} / \mathrm{Fe}]$} & $\ldots$ & $0.03 \pm 0.05$ & $\ldots$ \\
{$[\mathrm{Cr} / \mathrm{Fe}]$} & $0.01 \pm 0.02$ & $0.02 \pm 0.03$ & $\ldots$ \\
{$[\mathrm{Co} / \mathrm{Fe}]$} & $\ldots$ & $-0.01 \pm 0.04$ & $\ldots$ \\
{$[\mathrm{Ni} / \mathrm{Fe}]$} & $0.00 \pm 0.03$ & $-0.02 \pm 0.02$ & $-0.12 \pm 0.05$ \\
{$[\mathrm{Y} / \mathrm{Fe}]$} & $-0.05 \pm 0.06$ & $-0.10 \pm 0.09$ & $-0.12 \pm 0.09$ \\
{$[\mathrm{Ba} / \mathrm{Fe}]$} & $-0.02 \pm 0.14$ & $0.06 \pm 0.13$ & $-0.06 \pm 0.18$ \\
{$[\mathrm{Ce} / \mathrm{Fe}]$} & $\ldots$ & $0.01 \pm 0.15$ & $\ldots$ \\
{$[\mathrm{Nd} / \mathrm{Fe}]$} & $\ldots$ & $-0.06 \pm 0.10$ & $\ldots$ \\
{$[\mathrm{Eu} / \mathrm{Fe}]$} & $\ldots$ & $-0.02 \pm 0.11$ & $\ldots$ \\
\hline
\end{tabular}

Notes. Mean differences and standard deviations of the main parameters and abundance ratios $[\mathrm{El} / \mathrm{Fe}]$ for 13 stars of Group 1 in common with Bensby et al. (2014, B14), 7 stars of Group 1 in common with Reddy et al. (2006, R06), and 8 thin-disc stars in common with Edvardsson et al. (1993, E93).

caused mainly by differences in the applied determination methods. We see that titanium and zirconium abundances determined using both neutral and ionised lines agree well and confirm the $\log g$ values determined using iron lines. Overall, our $[\mathrm{El} / \mathrm{Fe}]$ for the stars in common agree very well with those in other studies.

\section{Results and discussion}

The atmospheric parameters, $T_{\mathrm{eff}}, \log g, v_{\mathrm{t}},[\mathrm{Fe} / \mathrm{H}]$ and the abundances of 21 chemical elements relative to iron $[\mathrm{El} / \mathrm{Fe}]$ of the programme and comparison stars are presented in Tables 4 and 5. The number of lines and the line-to-line scatter $(\sigma)$ are presented as well.

The results are graphically displayed in Figs. 7 and 8. We display elemental abundance ratios of Group 1 stars together with data of thin-disc stars investigated here and in Papers I-III, as well as with results taken from other thin-disc studies (Edvardsson et al. 1993; Gratton \& Sneden 1994; Koch \& Edvardsson 2002; Bensby et al. 2005; Reddy et al. 2006; Zhang \& Zhao 2006; Brewer \& Carney 2006; Mashonkina et al. 2007; Mishenina et al. 2013). The chemical evolution models of the thin-disc were taken from Pagel \& Tautvaišienè $(1995,1997)$. The thin-disc stars from Edvardsson et al. (1993) and Zhang \& Zhao (2006) were selected using the membership probability evaluation method described by Trevisan et al. (2011), since their lists contained stars of other Galactic components as well. The same kinematic approach in assigning thin-disc membership was used in Bensby et al. (2005) and Reddy et al. (2006), which means that the thin-disc stars used for comparison are uniform in that respect.

One star in Group 1 is rich in elements predominantly produced in s-process. As shown in Table 5 and Figs. 6 and 8, HD 88446 has much stronger lines of elements predominantly produced in s-process and consequently much higher abundances of these elements. According to the definition of Beers $\&$ Christlieb (2005), HD 88446, with its $[\mathrm{Ba} / \mathrm{Fe}]=1.04$ and $[\mathrm{Ba} / \mathrm{Eu}]=0.70$, falls in the category of the s-process-rich stars.

The metallicity of Group 1 stars we investigated lie in a broad interval of $0.04 \geq[\mathrm{Fe} / \mathrm{H}] \geq-0.57$ with the average $[\mathrm{Fe} / \mathrm{H}]$ equal to $-0.20 \pm 0.14$ dex. Abundances of chemical elements are rather homogeneous and show similar overabundances of $\alpha$-elements and r-process-dominated chemical elements with respect to thin-disc stars, as we also found for the stars of GCS Group 2 and 3. This elemental abundance pattern has similar characteristics with that in the Galactic thick-disc.

\subsection{Comparison with the thick-disc}

In Table 6 we present a comparison of mean $[\mathrm{El} / \mathrm{Fe}]$ ratios calculated for stars of Group 1 and thick-disc stars at the same metallicity interval $-0.57<[\mathrm{Fe} / \mathrm{H}]<0.04$. Twenty-six thickdisc stars in this metallicity interval were investigated by Bensby et al. (2005), 37 stars by Reddy et al. (2006), 10 stars by Mashonkina et al. (2007), 51 stars by Stanford \& Lambert (2012), and 7 stars by Mishenina et al. (2013). When comparing oxygen abundances, we did not use the results reported by Reddy et al. (2006) and Stanford \& Lambert (2012) because they investigated the O I line, while we studied [O I]. The studies by 
R. Ženovienè et al.: Stellar substructures in the solar neighbourhood. IV.

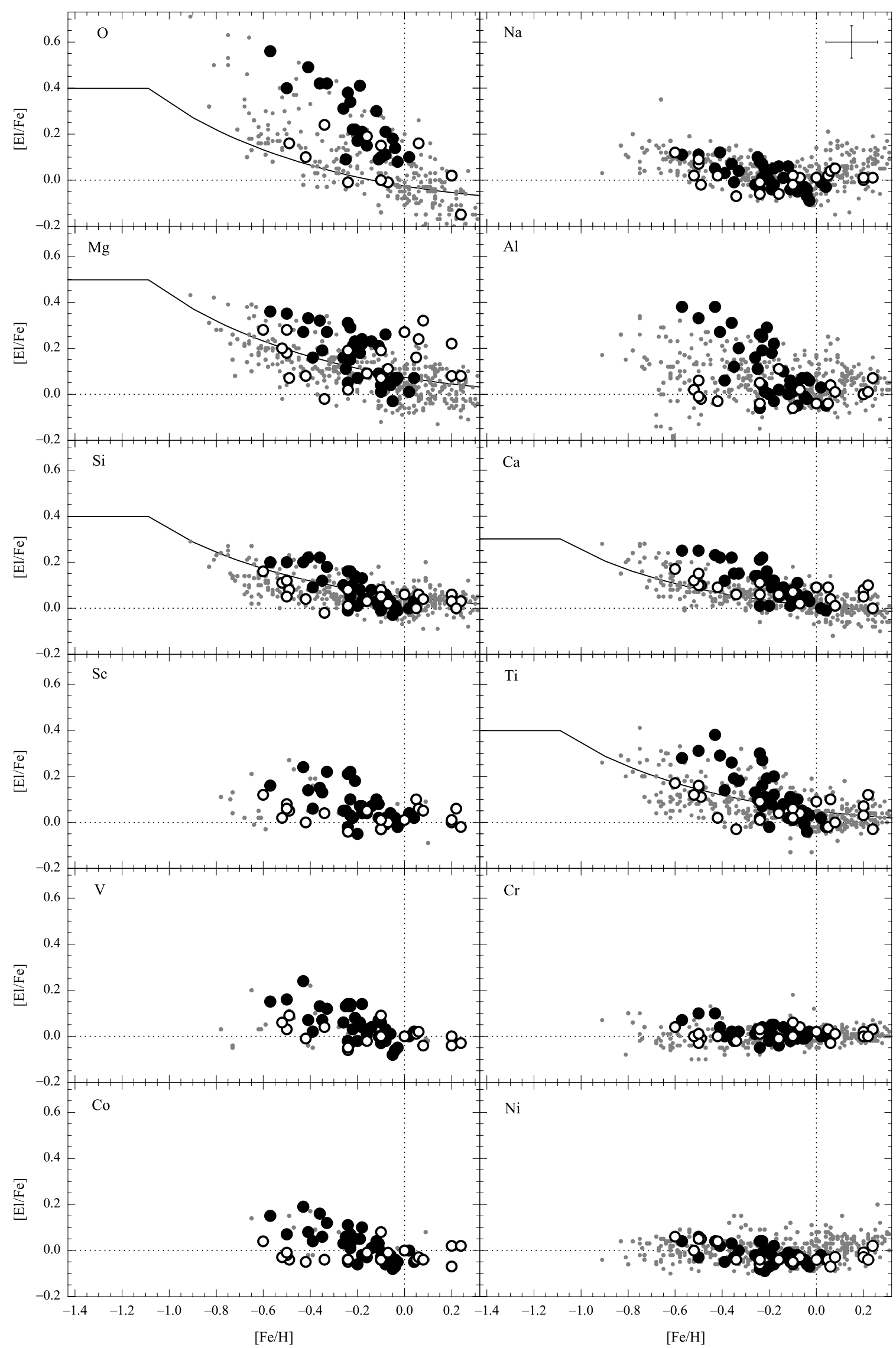

Fig. 7. $[\mathrm{El} / \mathrm{Fe}]$ ratio as a function of $[\mathrm{Fe} / \mathrm{H}]$ for Group 1 stars (filled circles) investigated here and for comparison thin-disc stars analysed in this work, Paper I, and Paper III (open circles). The data for the Milky Way thin-disc dwarfs taken from other studies are shown as a grey dots. Solid lines are Galactic thin-disc chemical evolution models presented by Pagel \& Tautvaišienè (1995). Average uncertainties are shown in the box for $\mathrm{Na}$. 


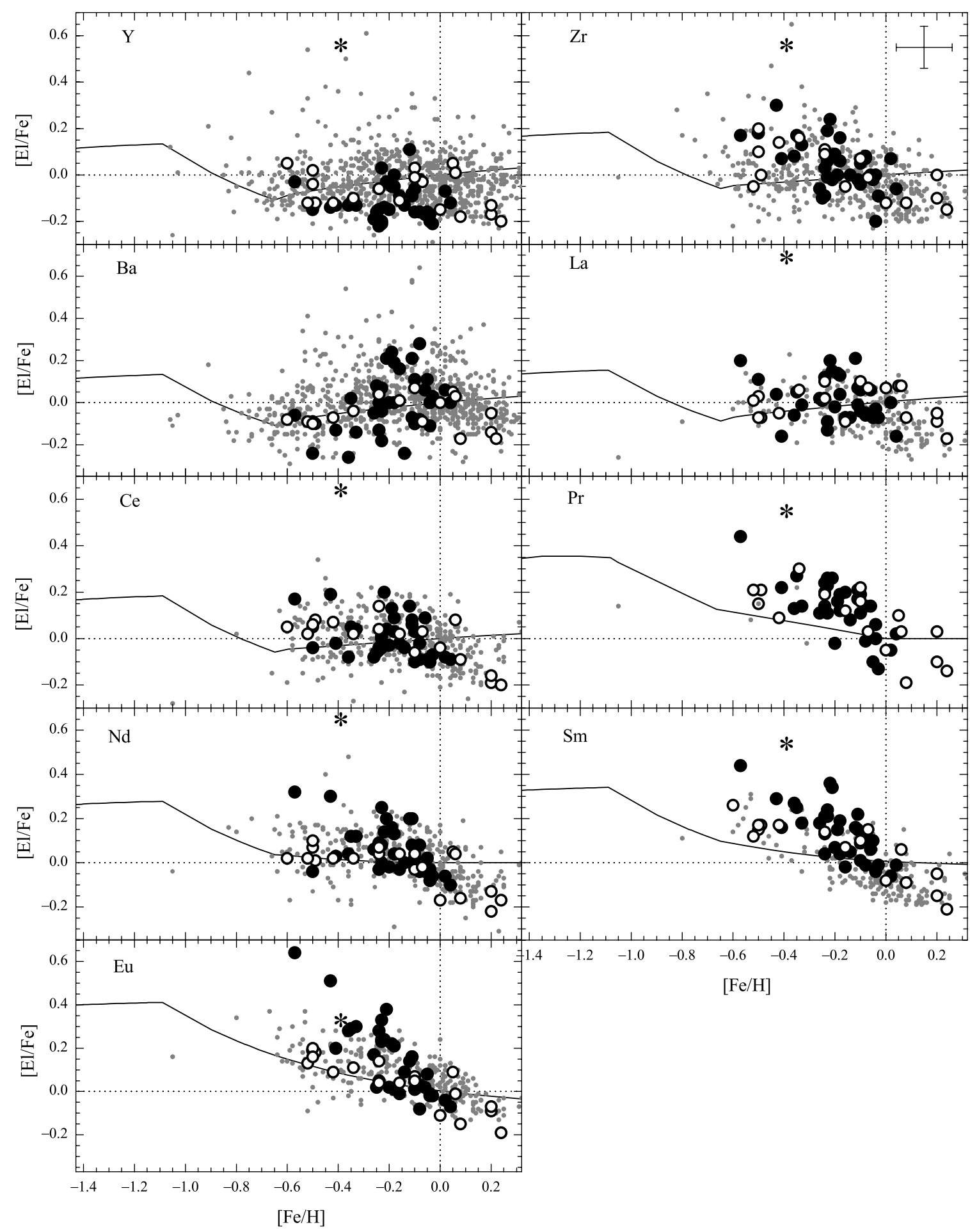

Fig. 8. $[\mathrm{El} / \mathrm{Fe}]$ ratio as a function of $[\mathrm{Fe} / \mathrm{H}]$ for Group 1 stars (filled circles) investigated here and for comparison thin-disc stars analysed in this work, Paper II, and Paper III (open circles). The s-process enhanced star (HD 88446) is marked as an asterisk. Grey dots correspond to the data for the Milky Way thin-disc dwarfs taken from other studies. The Galactic thin-disc chemical evolution model is shown as a solid line (Pagel \& Tautvaišienè 1997). Average uncertainties are shown in the box for Zr.

Mashonkina et al. (2007) and Mishenina et al. (2013) were included in the comparison to enlarge the information on neutron capture elements. The average values of $\alpha$-element abundances included $\mathrm{Mg}, \mathrm{Si}$, and $\mathrm{Ca}$. Titanium was excluded because this element was not determined in one of the studies (Mishenina et al. 2013). The comparison shows that the deviations do not exceed the uncertainties.
Figure 9 displays the comparison of $[\mathrm{El} / \mathrm{Fe}]$ ratios for some chemical elements between individual stars in Groups 1, 2, and 3 and the thick-disc stars of the above-mentioned studies. For comparison, we selected oxygen, the averaged values for the $\alpha$-elements $\mathrm{Mg}, \mathrm{Si}$, and $\mathrm{Ca}$, and the s- and r-process-dominated elements barium and europium. Stars of the kinematic groups and of the thick disc have very similar chemical compositions. 
Table 6. Comparison with thick-disc studies.

\begin{tabular}{lccccc}
\hline \hline$[\mathrm{El} / \mathrm{Fe}]$ & Ours-B14 & Ours-R06 & Ours-Ma07 & Ours-S12 & Ours-Mi13 \\
\hline$[\mathrm{O} / \mathrm{Fe}]$ & -0.02 & $\ldots$ & $\ldots$ & $\ldots$ & 0.08 \\
{$[\mathrm{Na} / \mathrm{Fe}]$} & -0.02 & -0.06 & $\ldots$ & -0.02 & $\ldots$ \\
{$[\mathrm{Mg} / \mathrm{Fe}]$} & -0.03 & -0.06 & $\ldots$ & -0.01 & -0.03 \\
{$[\mathrm{Al} / \mathrm{Fe}]$} & -0.07 & -0.12 & $\ldots$ & -0.07 & $\ldots$ \\
{$[\mathrm{Si} / \mathrm{Fe}]$} & -0.04 & -0.10 & $\ldots$ & -0.10 & -0.06 \\
{$[\mathrm{Ca} / \mathrm{Fe}]$} & 0.01 & -0.02 & $\ldots$ & -0.02 & -0.04 \\
{$[\mathrm{Sc} / \mathrm{Fe}]$} & $\ldots$ & -0.06 & $\ldots$ & -0.11 & $\ldots$ \\
{$[\mathrm{Ti} / \mathrm{Fe}]$} & -0.05 & -0.04 & $\ldots$ & -0.01 & $\ldots$ \\
{$[\mathrm{V} / \mathrm{Fe}]$} & $\ldots$ & -0.06 & $\ldots$ & -0.04 & $\ldots$ \\
{$[\mathrm{Cr} / \mathrm{Fe}]$} & 0.01 & 0.03 & $\ldots$ & -0.05 & $\ldots$ \\
{$[\mathrm{Co} / \mathrm{Fe}]$} & $\ldots$ & -0.06 & $\ldots$ & -0.05 & $\ldots$ \\
{$[\mathrm{Ni} / \mathrm{Fe}]$} & -0.03 & -0.05 & $\ldots$ & -0.02 & -0.05 \\
{$[\mathrm{Y} / \mathrm{Fe}]$} & -0.09 & -0.08 & -0.13 & -0.11 & -0.11 \\
{$[\mathrm{Zr} / \mathrm{Fe}]$} & $\ldots$ & $\ldots$ & -0.07 & $\ldots$ & 0.02 \\
{$[\mathrm{Ba} / \mathrm{Fe}]$} & 0.05 & 0.12 & 0.11 & 0.07 & 0.06 \\
{$[\mathrm{La} / \mathrm{Fe}]$} & $\ldots$ & $\ldots$ & $\ldots$ & $\ldots$ & 0.10 \\
{$[\mathrm{Ce} / \mathrm{Fe}]$} & $\ldots$ & -0.07 & -0.06 & -0.08 & 0.02 \\
{$[\mathrm{Nd} / \mathrm{Fe}]$} & $\ldots$ & -0.09 & $\ldots$ & -0.06 & -0.05 \\
{$[\mathrm{Sm} / \mathrm{Fe}]$} & $\ldots$ & $\ldots$ & $\ldots$ & $\ldots$ & 0.02 \\
{$[\mathrm{Eu} / \mathrm{Fe}]$} & $\ldots$ & -0.14 & $\ldots$ & -0.17 & -0.06 \\
\hline
\end{tabular}

Notes. Differences of mean $[\mathrm{El} / \mathrm{Fe}]$ values for stars of Group 1 and thick-disc stars at the same metallicity interval $-0.57<[\mathrm{Fe} / \mathrm{H}]<0.04$. Sixty-three stars from Bensby et al. (2014, B14), 37 stars from Reddy et al. (2006, R06), 10 stars from Mashonkina et al. (2007, Ma07), 51 stars from Stanford \& Lambert (2012, S12), and 10 stars from Mishenina et al. (2013, Mi13).

We have observed and analysed several thick-disc stars as well. Their elemental abundance ratios are also plotted in Fig. 9 and agree well with results of the programme stars. Thus, the chemical composition of all three GCS kinematic groups is similar to the thick-disc stars, which might suggest that their formation histories are linked.

\subsection{Age}

According to Helmi et al. (2006), the stars in Group 1 fall into two age populations: $33 \%$ of the stars are $8 \mathrm{Gyr}$ old, and $67 \%$ are 12 Gyr old. The ages were later redetermined by Holmberg et al. (2009) and Casagrande et al. (2011) and agree with each other within uncertainties (we present them in Table 1). Holmberg et al. (2009) present upper and lower age limits for every star. The average lower and upper limit is 10 and $14 \mathrm{Gyr}$ for stars of the 12 Gyr age population, 7 and 10 Gyr for the 8 Gyr population, and 4 and 5 Gyr for the younger stars, respectively. Figure 10 shows the Group 1 stars investigated here with our spectroscopic effective temperatures and absolute magnitudes $M_{v}$, taken from Holmberg et al. (2009), in a Hertzsprung-Russell (HR) diagram. The isochrones were taken from Bressan et al. (2012). The overall features of stars in the diagrams are well reproduced by isochrones of the two indicated ages. The more metal-abundant stars fit the 8 Gyr isochrone quite well, while more metal-deficient stars fit the 12 Gyr isochrone (for metaldeficient stars, the isochrones are with $[\alpha / \mathrm{Fe}]=0.2)$. A subgroup of ten stars in our sample are younger $(2 \mathrm{Gyr} \leq$ age $\leq$ 5 Gyr) and in the HR diagram lie higher than the turnoff luminosity of the $8 \mathrm{Gyr}$ isochrone. The number of stars younger than 5 Gyr among 120 stars in Group 1 is 18 (according to ages determined by Holmberg et al. 2009). A subgroup of about 15 young main-sequence stars can be separated among 86 Group 2 stars as well (Paper III). The chemical composition pattern of the young stars we investigated is similar to the rest of the GCS kinematic group stars of the same metallicity.

\subsection{Comparison with kinematic streams}

In this subsection, we discuss the GCS kinematic groups in the context of three other Galactic kinematic substructures of similar metallicities. Our attention was attracted by the Hercules stream. Stars of this kinematic stream have a similar range of metallicities and ages to those in the GCS groups (Bobylev \& Bajkova 2007; Antoja et al. 2008; Bensby et al. 2007, 2014). The Hercules stream was first identified by Eggen (1958) as a group of 22 stars with velocities similar to the high velocity star $\xi$ Herculis (HD 150680). It is believed that the Hercules stream is a result of resonant interactions between stars in the outer disc and the Galactic bar. This stellar stream is a heterogeneous group of objects from the thin and thick discs (Dehnen 2000; Fux 2001; Quillen 2003; Famaey et al. 2005; Soubiran \& Girard 2005; Pakhomov et al. 2011; Antoja et al. 2014; Bensby et al. 2014).

The origin of the Arcturus stream has been debated for years (Eggen 1971, 1996, 1998; Arifyanto \& Fuchs 2006; Gilmore et al. 2002; Wyse et al. 2006; Bensby et al. 2014, and references therein). Stars of the Arcturus stream were identified by Gilmore et al. (2002), and later Wyse et al. (2006), as a group of stars lagging behind the local standard of rest (LSR) by about $100 \mathrm{~km} \mathrm{~s}^{-1}$. This stream was associated with a disrupted satellite that merged with the Milky Way 10-12 Gyr ago. Navarro et al. (2004) suggested that these stars are the same group of stars that Eggen (1971) associated with the bright star Arcturus, whose Galactic orbital velocity also lags at the same value. Navarro et al. (2004) analysed the group of stars associated kinematically with Arcturus and confirmed that they constitute a peculiar grouping of metal-poor stars with a similar apocentric radius, a common angular momentum, and distinct metal abundance patterns. These properties are consistent with those expected for a group of stars originating from the debris of a disrupted satellite. Navarro et al. (2004) also noticed that the angular momentum of such a group is too low to arise from dynamical perturbations induced by the Galactic bar. More recently, Gardner \& Flynn (2010) and Monari et al. (2013) showed that the Galactic long bar may produce a kinematic feature in velocity space with the same parameters as occupied by the Arcturus moving group.

Another so-called AF06 stellar stream was discovered by Arifyanto \& Fuchs (2006) while analysing the fine structure of the phase space distribution function of nearby subdwarfs using data extracted from various catalogues. According to the discoverers, AF06 possibly resembles the Arcturus stream.

Figure 11 presents a Toomre diagram and velocity distributions of stars in the GCS kinematic groups and Hercules, Arcturus, and AF06 streams. This diagram shows that the kinematics of stars in the GCS groups is quite different from the displayed streams, and only AF06 partially overlaps the pattern.

A comparison of $[\mathrm{El} / \mathrm{Fe}]$ ratios for $\alpha$-elements between individual stars in the GCS groups and stars of the Arcturus, AF06, and Hercules streams is presented in Fig. 12. The averaged values for $\alpha$-elements consist of $\mathrm{Mg}, \mathrm{Si}, \mathrm{Ca}$, and Ti abundances. We took the elemental abundances for 33 stars of the Hercules stream from Soubiran \& Girard (2005) and for 35 stars from Bensby et al. (2014). We took the elemental abundances for 18 stars of the Arcturus stream and for 26 stars of the 


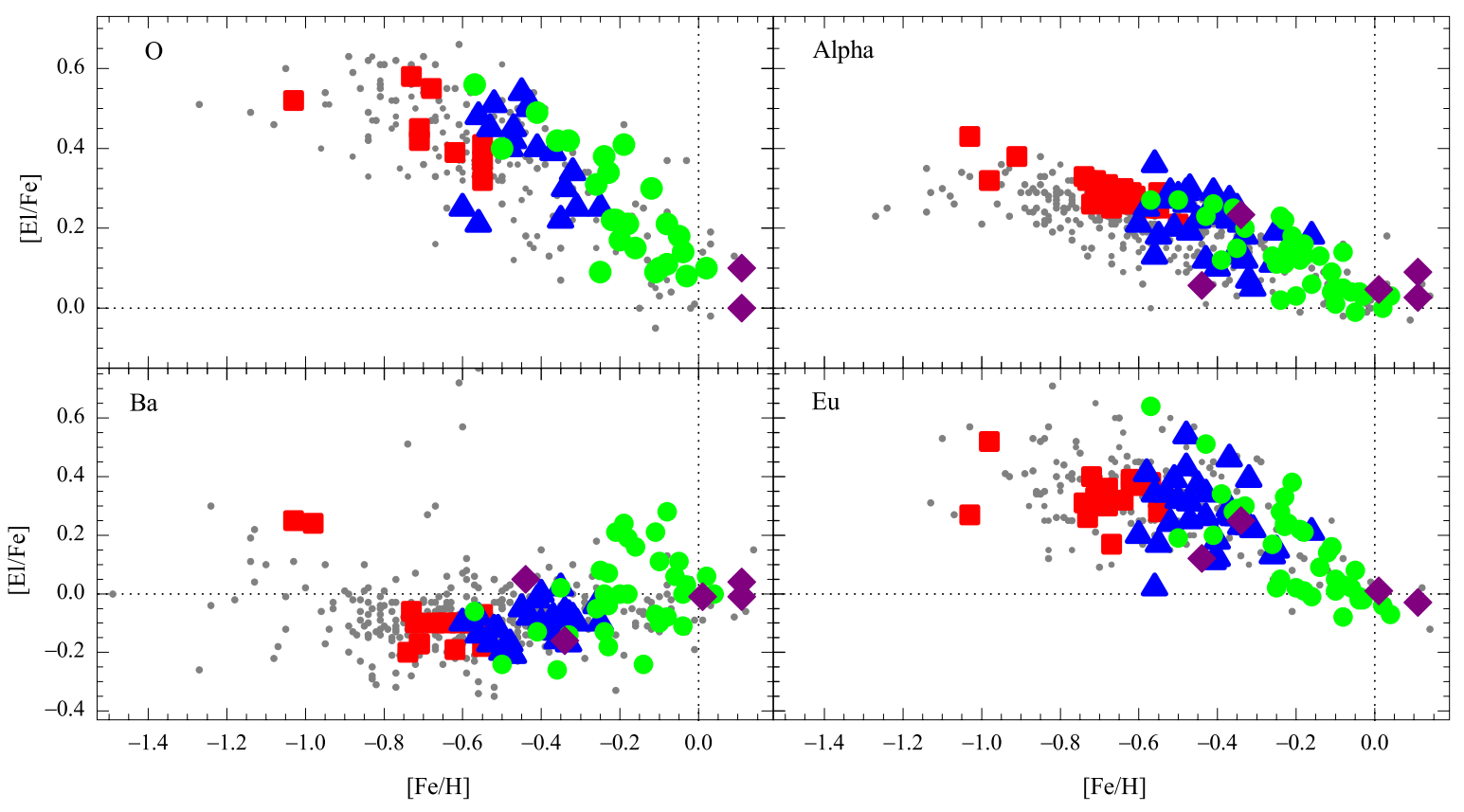

Fig. 9. $[\mathrm{El} / \mathrm{Fe}]$ ratio as a function of $[\mathrm{Fe} / \mathrm{H}]$ in the Group 1 stars (green dots) investigated here, Group 2 (blue triangles, Paper III), Group 3 (red squares, Papers I and II), and comparison thick-disc stars (purple diamonds). The literature data for the Milky Way thick-disc stars are shown as a grey dots. The averaged values for $\alpha$-elements consist of $\mathrm{Mg}$, $\mathrm{Si}$, and $\mathrm{Ca}$ abundances.

AF06 stream from Ramya et al. (2012). In Fig. 12 we also show the chemical evolution model of the Galactic thin-disc by Pagel \& Tautvaišienè (1995) and a simple second-order polynomial fit to the GCS kinematic group stars.

The element-to-iron ratios in the GCS kinematic stellar groups lie higher than in the majority of stars belonging to Arcturus, AF06, and Hercules streams. The AF06 group has the chemical composition most similar to the GCS stars. In Paper III, we pointed out that the Arcturus group has thick-disc kinematics, but it has seemingly thin-disc abundances. Finally, as noted previously, the stars associated with the Hercules stream do not have a distinct chemical signature, but show a mixture of abundances as seen in the thin and thick discs (cf. Soubiran \& Girard 2005; Bensby et al. 2007, 2014; Pakhomov et al. 2011).

Thus, the presented comparison of kinematic and chemical composition patterns in the Galactic substructures, leads us to the conclusion that the origin of the GCS kinematic stellar groups was different from Galactic streams that originated in a course of the resonant interactions between stars in the outer disc and the Galactic bar.

\subsection{Origin}

A sample of 274 stars was identified as an overdensity in the eccentricity range $0.3<\epsilon<0.5$ in the Geneva-Copenhagen survey by Helmi et al. (2006). The authors provide statistical evidence that these overdensities are real, and that they do not result from a poor choice of the comparison model of the Galaxy or uncertainties of eccentricity determinations. It was found that stars with these dynamical characteristics do not constitute a homogeneous population. The metallicity distribution of the stars in this overdense region of the APL-space varied with eccentricity in a discontinuous fashion. This allowed the authors to separate these stars into three kinematic groups. These three groups of stars are dissimilar not only in their metallicity distribution, but

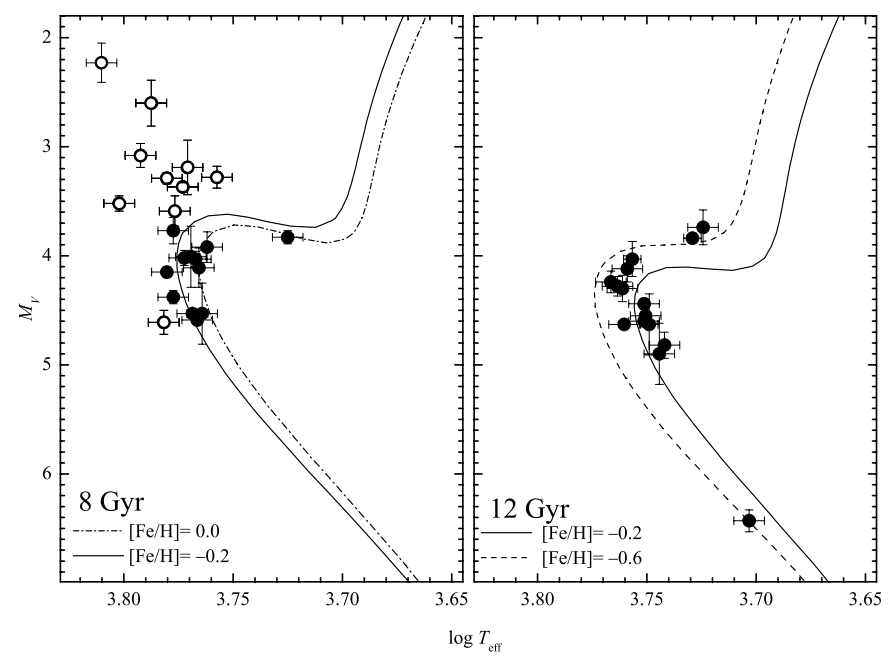

Fig. 10. HR diagrams of the Group 1 stars. Isochrones are taken from Bressan et al. (2012). The isochrones for metal-deficient stars are with $[\alpha / \mathrm{Fe}]=0.2$. The open circles represent the younger stars with ages of 2-5 Gyr.

they also have different kinematics in the vertical $(z)$ direction. The Group 1 velocity dispersion has $\sigma_{z}$ about $28 \mathrm{~km} \mathrm{~s}^{-1}$, that of Group 2 about $39 \mathrm{~km} \mathrm{~s}^{-1}$, and that of Group 3 about $52 \mathrm{~km} \mathrm{~s}^{-1}$.

Helmi et al. (2014) determined the detailed chemical composition for 36 stars of the GCS Group 1, for 22 stars of Group 2, and 14 stars of Group 3 located in the southern hemisphere. In this study, they noticed a relatively sharp transition in dynamical and chemical properties that occurs at a metallicity of $[\mathrm{Fe} / \mathrm{H}] \sim-0.4$. In their sample, stars with $[\mathrm{Fe} / \mathrm{H}]>-0.4$ have mostly lower eccentricities, smaller vertical velocity dispersions, are $\alpha$-enhanced, and define a rather narrow sequence in $[\alpha / \mathrm{Fe}]$ versus $[\mathrm{Fe} / \mathrm{H}]$, clearly distinct from that of the thin disc. Stars with $[\mathrm{Fe} / \mathrm{H}]<-0.4$ have a range of eccentricities, are hotter 
R. Ženovienè et al.: Stellar substructures in the solar neighbourhood. IV.
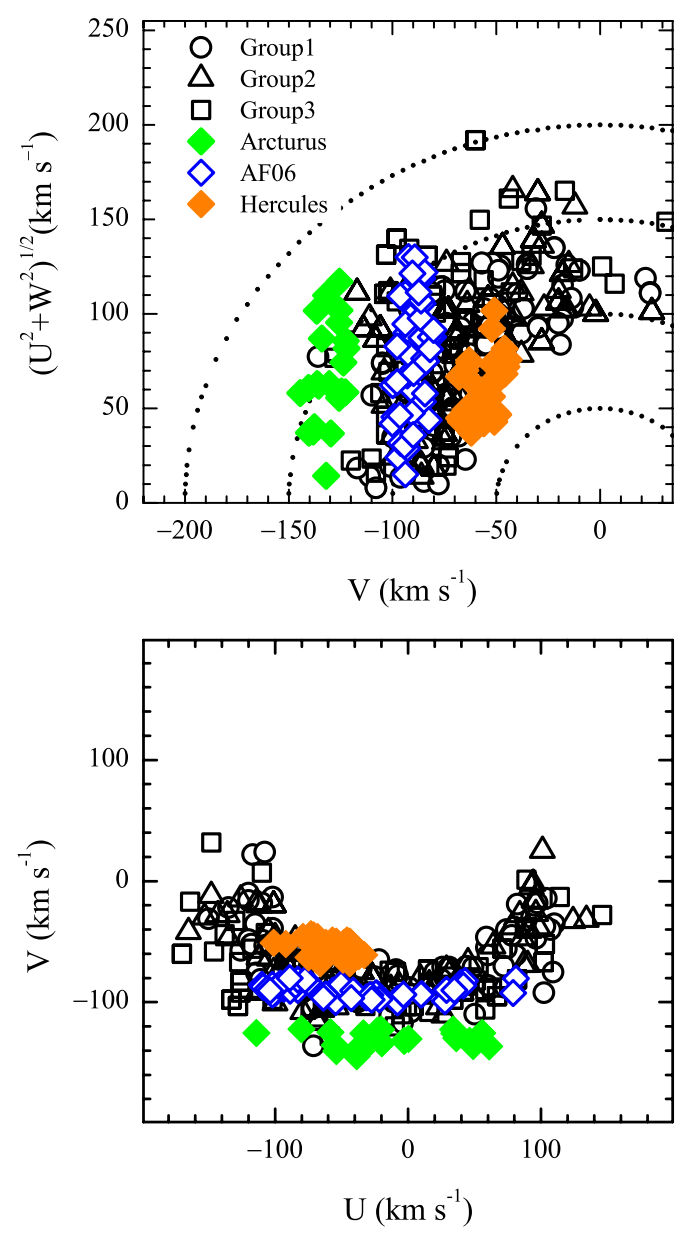

Fig. 11. Upper panel: toomre diagram of stars in GCS kinematic groups and Arcturus, AF06, and Hercules streams. Dotted lines indicate constant values of total space velocity in steps of $50 \mathrm{~km} \mathrm{~s}^{-1}$. Lower panel: velocity distribution for stars in the same kinematic groups and streams.

vertically, and depict a larger spread in $[\alpha / \mathrm{Fe}]$. Looking at our slightly larger sample of GCS kinematic group stars (Fig. 13) investigated here, we agree with Helmi et al. (2014) that stars with lower metallicities have a wider range of eccentricities than those that are more metal-abundant. However, the spread of $[\alpha / \mathrm{Fe}]$, seems to be about the same at all metallicities (see Figs. 9 and 12). In Fig. 13, we mark stars of different ages with different colours. Practically all stars with $[\mathrm{Fe} / \mathrm{H}]<-0.4$ have ages around $12 \mathrm{Gyr}$, while the youngest stars are predominantly metal-abundant.

Even though, according to the dynamical characteristics, the stars of the GCS kinematic groups do not constitute a homogeneous population, their similar chemical composition pattern indicates that these kinematic groups might share a similar origin. The similarity in chemical composition of stars in these kinematic groups and in the thick-disc of the Galaxy suggests that the formation histories of these groups and the thick-disc might be linked. This circumstance led us to look for the currently available thick-disc formation scenario, which allows for the presence of stars with GCS kinematic group characteristics. As we pointed out in Papers I-III, the kinematic properties of the GCS kinematic groups fit a gas-rich satellite merger scenario (Brook et al. 2004, 2005; Dierickx et al. 2010; Wilson et al. 2011; Di Matteo et al. 2011) best of all. Within this particular scenario, the eccentricities of accreted stars peak at about $0.3<\epsilon<0.5$ (Sales et al. 2009), which are exactly the characteristics of the GCS groups that we investigated. The gasrich merger scenario is worth considering since, according to Dierickx et al. (2010), it fits the thick-disc star eccentricity distribution better than the accretion, heating, or migration scenarios. Although it is likely that all of those processes to some extent acted in the Milky Way, it is not clear which, if any, was the dominant mechanism.

Finally, the numerical simulations of the disruption of a satellite galaxy that falls into its parent galaxy shows that the satellite debris can end up in several cold star streams with roughly the same characteristic eccentricities of their orbits (Helmi et al. 2006). The possibility of such a scheme and the similar properties of element-to-iron ratios found in the GCS kinematic groups lead to the assumption that the GCS kinematic star groups might belong to the same satellite galaxy and might have originated in our Galaxy during the same merging event.

Investigations of formation and evolution of the Milky Way discs are continuing both observationally (e.g. Haywood et al. 2013; Kordopatis et al. 2013b; Anders et al. 2014; Bensby et al. 2014; Bergemann et al. 2014; Mikolaitis et al. 2014) and theoretically (Micali et al. 2013; Snaith et al. 2014; Robin et al. 2014; Kubryk et al. 2014; Minchev et al. 2014, and references therein). A model that could reproduce well the present day values of all of the main global observables of the Milky Way disc has not been discovered yet.

\section{Conclusions}

We measured abundances of 22 chemical elements from highresolution spectra in 37 stars belonging to Group 1 of the Geneva-Copenhagen survey. This kinematically identified group of stars as well as two other GCS kinematic groups was suggested to be a remnant of a disrupted satellite galaxy. Our main goal was to investigate the chemical composition of stars within Group 1, to compare it with the relative abundance patterns in the Galactic thin- and thick-disc stars, in the GCS Group 2 and Group 3 stars, as well as in several kinematic streams of similar metallicities.

Our study shows the following:

1. The metallicities of the investigated stars in Group 1 are in the range of $0.04 \geq[\mathrm{Fe} / \mathrm{H}] \geq-0.57$. The average $[\mathrm{Fe} / \mathrm{H}]$ value is $-0.20 \pm 0.14 \mathrm{dex}$.

2. Investigated Group 1 stars can be separated into three age subgroups. Along with the main 8- and 12-Gyr-old populations, a subgroup of stars younger than 5 Gyr can be separated as well.

3. All programme stars have higher abundances in oxygen, $\alpha$ elements, and r-process-dominated chemical elements than Galactic thin-disc dwarfs and the Galactic evolution model. The abundances of iron-group chemical elements and elements produced mainly by the s-process are similar to those in the Galactic thin-disc dwarfs of the same metallicity.

4. The chemical composition and kinematic properties in the GCS Group 1, 2, and 3 stars are different from those in stars of the Hercules, Arcturus and AF06 streams.

5. The chemical composition patterns in GCS Groups 1,2, and 3 are similar to each other and to the thick-disc stars, which might suggest that their formation histories are linked.

6. The chemical composition together with the kinematic properties and ages of stars in the investigated GCS Group 1,2 


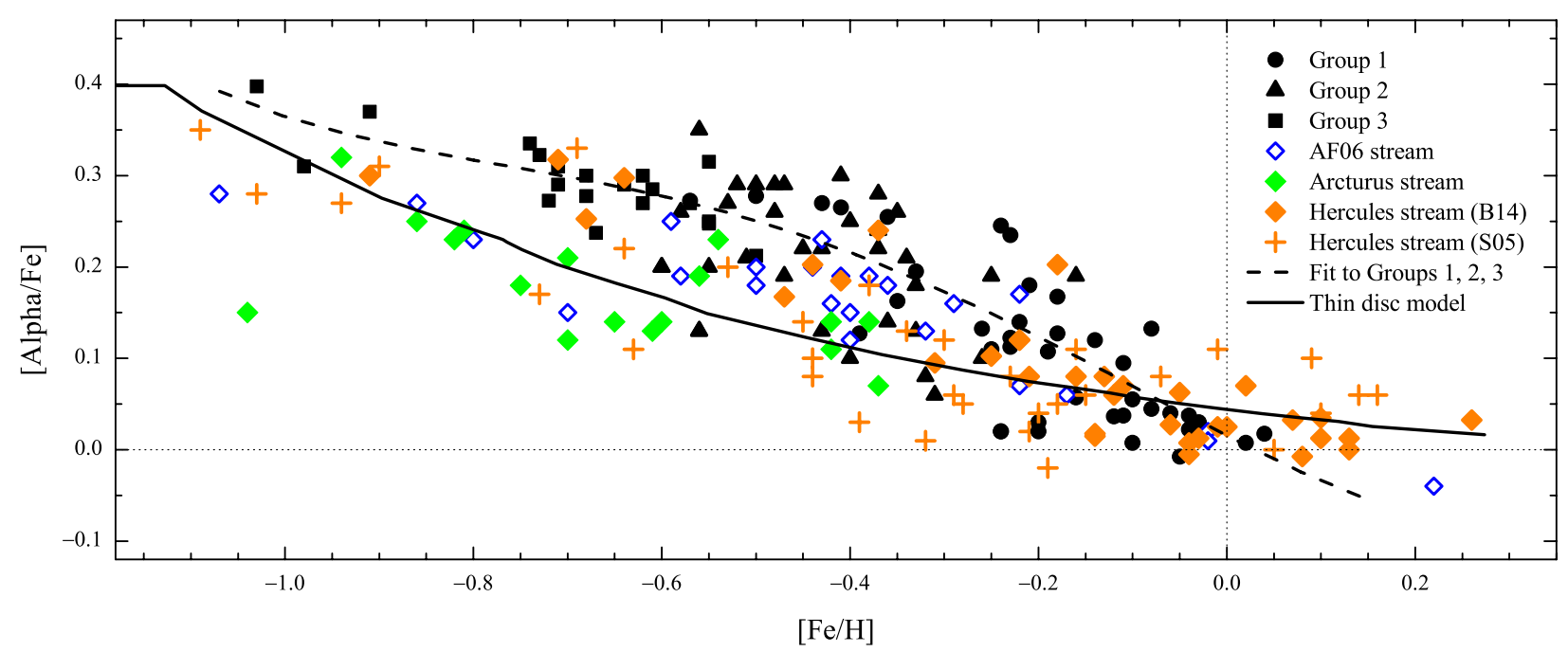

Fig. 12. $[\alpha / \mathrm{Fe}]$ ratio as a function of $[\mathrm{Fe} / \mathrm{H}]$ in the GCS kinematic group of stars, in the Arcturus stream (green diamonds), in the AF06 stream (blue empty diamonds), and in the Hercules stream (orange diamonds and crosses). The data for Arcturus and AF06 streams were taken from Ramya et al. (2012), for the Hercules stream from Soubiran \& Girard (2005) and Bensby et al. (2014). The averaged values for $\alpha$-elements consist of $\mathrm{Mg}, \mathrm{Si}, \mathrm{Ca}$, and Ti abundances. A thin-disc model (continuous line) is taken from Pagel \& Tautvaišienè (1995), the 2nd order polynomial fit to the GCS data is shown by the dashed line.

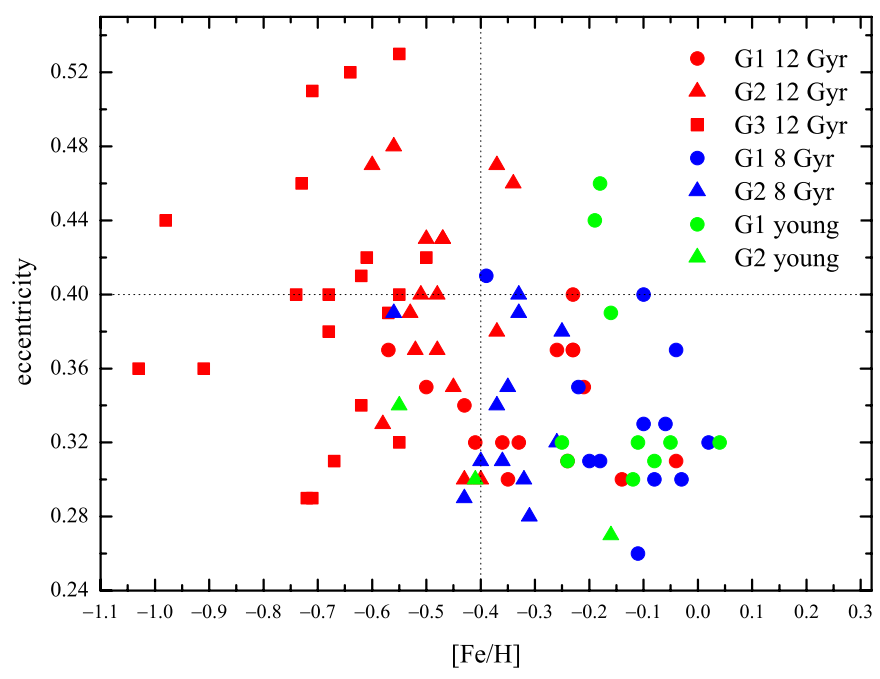

Fig. 13. Eccentricity and $[\mathrm{Fe} / \mathrm{H}]$ diagram of the GCS kinematic group stars. The red symbols correspond to stars investigated here with ages of about $12 \mathrm{Gyr}$, blue to about $8 \mathrm{Gyr}$, and green to the younger stars in Groups 1, 2, and 3 .

and 3 support a gas-rich satellite merger scenario as a possible origin for these kinematic groups.

Acknowledgements. The data are based on observations made with the Nordic Optical Telescope, operated on the island of La Palma jointly by Denmark, Finland, Iceland, Norway, and Sweden, in the Spanish Observatorio del Roque de los Muchachos of the Instituto de Astrofisica de Canarias. The research leading to these results has received funding from the European Community's Seventh Framework Programme (FP7/2007-2013) under grant agreement number RG226604 (OPTICON). B.N. acknowledges support from the Danish Research council and the Carlsberg Foundation. This research has made use of SIMBAD, VALD, and NASA ADS databases.

\section{References}

Anders, F., Chiappini, C., Santiago, B. X., et al. 2014, A\&A, 564, A115 Antoja, T., Figueras, F., Fernández, D., \& Torra, J. 2008, A\&A, 490, 135 Antoja, T., Helmi, A., Bienayme, O., et al. 2012, MNRAS, 426, 1

Antoja, T., Helmi, A., Dehnen, W., et al. 2014, A\&A, 563, A60

Arifyanto, M. I., \& Fuchs, B. 2006, A\&A, 449, 533
Beers, T. C., \& Christlieb, N. 2005, ARA\&A, 43, 531

Bensby, T., Feltzing, S., Lundström, I., \& Ilyin, I. 2005, A\&A, 433, 185 Bensby, T., Oey, M. S., Feltzing, S., \& Gustafsson, B. 2007, ApJ, 655, L89

Bensby, T., Feltzing, S., \& Oey, M. S. 2014, A\&A, 562, A71

Bergemann, M., Ruchti, G. R., Serenelli, A., et al. 2014, A\&A, 565, A89

Bobylev, V. V., \& Bajkova, A. T. 2007, Astron. Rep., 51, 372

Bobylev, V. V., Bajkova, A. T., \& Mylläri, A. A. 2010, Astron. Lett., 36, 27

Bressan, A., Marigo, P., Girardi, L., et al. 2012, MNRAS, 427, 127

Brewer, M.-M., \& Carney, B. W. 2006, AJ, 131, 431

Brook, C. B., Kawata, D., Gibson, B. K., \& Freeman, K. C. 2004, ApJ, 612, 894

Brook, C. B., Gibson, B. K., Martel, H., \& Kawata, D. 2005, ApJ, 630, 298 Casagrande, L., Schönrich, R., Asplund, M., et al. 2011, A\&A, 530, A138 Dehnen, W. 2000, AJ, 119, 800

Dekker, E. 1976, Phys. Rep., 24, 315

Dettbarn, C., Fuchs, B., Flynn, C., \& Williams, M. 2007, A\&A, 474, 857

Di Matteo, P., Lehnert, M. D., Qu, Y., \& van Driel, W. 2011, A\&A, 525, L3

Dierickx, M., Klement, R., Rix, H.-W., \& Liu, C. 2010, ApJ, 725, L186

Edvardsson, B., Andersen, J., Gustafsson, B., et al. 1993, A\&A, 275, 101

Eggen, O. J. 1958, MNRAS, 118, 154

Eggen, O. J. 1971, PASP, 83, 271

Eggen, O. J. 1996, AJ, 112, 1595

Eggen, O. J. 1998, AJ, 115, 2397

Famaey, B., Jorissen, A., Luri, X., et al. 2005, A\&A, 430, 165

Flynn, C., Sommer-Larsen, J., \& Christensen, P. R. 1996, MNRAS, 281, 1027

Fux, R. 2001, A\&A, 373, 511

Gardner, E., \& Flynn, C. 2010, MNRAS, 405, 545

Gilmore, G., \& Reid, N. 1983, MNRAS, 202, 1025

Gilmore, G., Wyse, R. F. G., \& Norris, J. E. 2002, ApJ, 574, L39

Gómez, F. A., Minchev, I., Villalobos, Á., O’Shea, B. W., \& Williams, M. E. K. 2012, MNRAS, 419, 2163

Gratton, R. G., \& Sneden, C. 1994, A\&A, 287, 927

Grevesse, N., \& Sauval, A. J. 2000, Origin of Elements in the Solar System, Implications of Post-1957 Observations, 261

Gurtovenko, E. A., \& Kostyk, R. I. 1989, Kiev Izdatel Naukova Dumka

Gustafsson, B., Edvardsson, B., Eriksson, K., et al. 2008, A\&A, 486, 951

Haywood, M., Di Matteo, P., Lehnert, M. D., Katz, D., \& Gómez, A. 2013, A\&A, 560, A 109

Helmi, A. 2004, ApJ, 610, L97

Helmi, A., Navarro, J. F., Nordström, B., et al. 2006, MNRAS, 365, 1309

Helmi, A., Williams, M., Freeman, K. C., Bland-Hawthorn, J., \& De Silva, G. 2014, ApJ, 791, 135

Holmberg, J., Nordström, B., \& Andersen, J. 2009, A\&A, 501, 941

Ilyin, I. V. 2000, Ph.D. dissertation, Univ. Oulu, Finland

Kirby, E. N., Lanfranchi, G. A., Simon, J. D., Cohen, J. G., \& Guhathakurta, P. 2011, ApJ, 727, 78

Klement, R. 2010, A\&ARv, 18, 567 
R. Ženovienè et al.: Stellar substructures in the solar neighbourhood. IV.

Klement, R, Fuchs, B , \& Rix, H.-W. 2008, ApJ, 685, 261

Klement, R., Rix, H.-W., Flynn, C., et al. 2009, ApJ, 698, 865

Klement, R. J., Bailer-Jones, C. A. L., Fuchs, B., Rix, H.-W., \& Smith, K. W. 2011, ApJ, 726, 103

Koch, A., \& Edvardsson, B. 2002, A\&A, 381, 500

Kordopatis, G., Hill, V., Irwin, M., et al. 2013a, A\&A, 555, A12

Kordopatis, G., Gilmore, G., Wyse, R. F. G., et al. 2013b, MNRAS, 436, 3231

Kubryk, M., Prantzos, N., \& Athanassoula, E. 2014, A\&A, submitted [arXiv: 1412.0585]

Kurucz, R. L. 2005, Memo. Soc. Astron. It. Suppl., 8, 189

Law, D. R., Johnston, K. V., \& Majewski, S. R. 2005, ApJ, 619, 807

Mashonkina, L. I., Vinogradova, A. B., Ptitsyn, D. A., Khokhlova, V. S., \& Chernetsova, T. A. 2007, Astron. Rep., 51, 903

Micali, A., Matteucci, F., \& Romano, D. 2013, MNRAS, 436, 1648

Mikolaitis, Š., Hill, V., Recio-Blanco, A., et al. 2014, A\&A, 572, A33

Minchev, I., Quillen, A. C., Williams, M., et al. 2009, MNRAS, 396, L56

Minchev, I., Chiappini, C., \& Martig, M. 2014, A\&A, 572, A92

Mishenina, T. V., Pignatari, M., Korotin, S. A., et al. 2013, A\&A, 552, A128

Monari, G., Antoja, T., \& Helmi, A. 2013 [arXiv: 1306 . 2632]

Navarro, J. F., Helmi, A., \& Freeman, K. C. 2004, ApJ, 601, L43

Nordström, B., Mayor, M., Andersen, J., et al. 2004, A\&A, 418, 989

Pagel, B. E. J., \& Tautvaišienè, G. 1995, MNRAS, 276, 505

Pagel, B. E. J., \& Tautvaišienè, G. 1997, MNRAS, 288, 108

Pakhomov, Y. V., Antipova, L. I., \& Boyarchuk, A. A. 2011, Astron. Rep., 55, 256
Pauli, E-M., Napiwotzki, R., Altmann, M., et al. 2003, A\&A, 400, 877 Peñarrubia, J., Martínez-Delgado, D., Rix, H. W., et al. 2005, ApJ, 626, 128

Perryman, M. A. C., de Boer, K. S., Gilmore, G., et al. 2001, A\&A, 369, 339

Piskunov, N. E., Kupka, F., Ryabchikova, T. A., Weiss, W. W., \& Jeffery, C. S. 1995, A\&AS, 112, 525

Quillen A. C. 2003, AJ, 125, 785

Ramya, P., Reddy, B. E., \& Lambert, D. L. 2012, MNRAS, 425, 3188

Reddy, B. E., Lambert, D. L., \& Allende Prieto, C. 2006, MNRAS, 367, 1329

Robin, A. C., Reylé, C., Fliri, J., et al. 2014, A\&A, 569, A13

Sales, L. V., Helmi, A., Abadi, M. G., et al. 2009, MNRAS, 400, L61

Snaith, O. N., Haywood, M., Di Matteo, P., et al. 2014, ApJ, 781, L31

Stanford, L. M., \& Lambert, D. L. 2012, MNRAS, 424, 2118

Stonkute, E., Tautvaišienė, G., Nordström, B., \& Ženovienė, R. 2012, A\&A, 541 , A157 (Paper I)

Stonkutè, E., Tautvaišienė, G., Nordström, B., \& Ženovienė, R. 2013, A\&A, 555 A6 (Paper II)

Soubiran, C., \& Girard, P. 2005, A\&A, 438, 139

Trevisan, M., Barbuy, B., Eriksson, K., et al. 2011, A\&A, 535, A42

Villalobos, Á., \& Helmi, A. 2008, MNRAS, 391, 1806

Villalobos, Á., \& Helmi, A. 2009, MNRAS, 399, 166

Wilson, M. L., Helmi, A., Morrison, H. L., et al. 2011, MNRAS, 413, 2235

Wu, Z.-Y., Zhou, X., Ma, J., \& Du, C.-H. 2009, MNRAS, 399, 2146

Wyse, R. F. G., Gilmore, G., Norris, J. E., et al. 2006, ApJ, 639, L13

Ženovienė, R., Tautvaišienė, G., Nordström, B., \& Stonkutė, E. 2014, A\&A, 563, A53 (Paper III)

Zhang, H. W., \& Zhao, G. 2006, A\&A, 449, 127 
A\&A 576, A113 (2015)

Table 4. Main atmospheric parameters and elemental abundances of programme and comparison stars.

\begin{tabular}{|c|c|c|c|c|c|c|c|c|c|c|c|c|c|c|c|}
\hline Star & $\begin{array}{c}T_{\text {eff }} \\
\mathrm{K}\end{array}$ & $\log g$ & $\begin{array}{c}v_{\mathrm{t}} \\
\mathrm{km} \mathrm{s}^{-1}\end{array}$ & {$[\mathrm{Fe} / \mathrm{H}]$} & $\sigma_{\mathrm{FeI}}$ & $n_{\mathrm{FeI}}$ & $\sigma_{\mathrm{FeII}}$ & $n_{\mathrm{FeII}}$ & {$[\mathrm{O} / \mathrm{Fe}]$} & {$[\mathrm{Na} / \mathrm{Fe}]$} & $\sigma$ & $n$ & {$[\mathrm{Mg} / \mathrm{Fe}]$} & $\sigma$ & $n$ \\
\hline \multicolumn{16}{|l|}{ Group 1 stars } \\
\hline HD 3795 & 5360 & 3.7 & 1.0 & -0.57 & 0.05 & 31 & 0.04 & 6 & 0.56 & 0.11 & 0.02 & 4 & 0.36 & 0.05 & 4 \\
\hline HD 4607 & 6200 & 3.8 & 1.2 & -0.08 & 0.05 & 25 & 0.03 & 6 & 0.21 & 0.00 & 0.02 & 2 & 0.26 & 0.00 & 3 \\
\hline HD 15777 & 5800 & 4.2 & 1.0 & -0.33 & 0.04 & 28 & 0.04 & 6 & 0.42 & 0.04 & 0.01 & 5 & 0.27 & 0.06 & 3 \\
\hline HD 22872 & 5980 & 4.0 & 1.1 & 0.04 & 0.05 & 34 & 0.02 & 8 & & -0.03 & 0.01 & 3 & 0.07 & 0.09 & 4 \\
\hline HD 25123 & 5880 & 3.9 & 1.1 & 0.02 & 0.04 & 37 & 0.06 & 8 & 0.10 & -0.01 & 0.01 & 5 & 0.01 & 0.02 & 3 \\
\hline HD 40040 & 5740 & 4.0 & 1.1 & -0.24 & 0.05 & 37 & 0.05 & 7 & 0.38 & 0.08 & 0.02 & 3 & 0.31 & 0.06 & 4 \\
\hline HD 49409 & 5770 & 4.1 & 0.9 & -0.23 & 0.05 & 32 & 0.04 & 6 & $\ldots$ & 0.01 & 0.02 & 3 & 0.15 & 0.05 & 4 \\
\hline HD 52711 & 5870 & 4.1 & 1.0 & -0.08 & 0.05 & 38 & 0.03 & 7 & 0.11 & -0.05 & 0.01 & 4 & 0.06 & 0.05 & 4 \\
\hline HD 60779 & 5990 & 4.1 & 0.9 & -0.10 & 0.05 & 36 & 0.04 & 8 & 0.10 & 0.02 & 0.03 & 4 & 0.04 & 0.03 & 3 \\
\hline HD 67088 & 5610 & 4.0 & 0.8 & -0.04 & 0.04 & 37 & 0.03 & 7 & 0.14 & -0.07 & 0.04 & 5 & 0.07 & 0.05 & 4 \\
\hline HD 67587 & 6030 & 3.8 & 1.1 & -0.25 & 0.05 & 34 & 0.05 & 8 & 0.09 & 0.10 & 0.03 & 4 & 0.11 & 0.03 & 3 \\
\hline HD 76095 & 5720 & 4.1 & 1.0 & -0.19 & 0.04 & 35 & 0.05 & 8 & 0.41 & -0.04 & 0.01 & 4 & 0.18 & 0.05 & 4 \\
\hline HD 77408 & 6340 & 4.2 & 1.1 & -0.11 & 0.04 & 25 & 0.04 & 7 & $\ldots$ & -0.04 & 0.05 & 3 & 0.21 & 0.11 & 3 \\
\hline HD 78558 & 5640 & 4.0 & 0.9 & -0.41 & 0.05 & 36 & 0.04 & 7 & 0.49 & 0.12 & 0.06 & 5 & 0.33 & 0.07 & 4 \\
\hline HD 88371 & 5630 & 4.2 & 0.8 & -0.23 & 0.04 & 38 & 0.06 & 8 & 0.34 & 0.01 & 0.05 & 5 & 0.29 & 0.06 & 4 \\
\hline HD 88446 & 5990 & 3.9 & 1.2 & -0.39 & 0.05 & 34 & 0.03 & 8 & $\ldots$ & 0.03 & 0.04 & 3 & 0.16 & 0.04 & 3 \\
\hline HD 90508 & 5760 & 4.1 & 1.0 & -0.26 & 0.04 & 37 & 0.05 & 8 & 0.31 & -0.02 & 0.06 & 5 & 0.16 & 0.01 & 4 \\
\hline HD 109498 & 5810 & 4.2 & 1.0 & -0.11 & 0.06 & 30 & 0.06 & 6 & 0.09 & 0.01 & 0.06 & 4 & 0.09 & 0.02 & 3 \\
\hline HD 111367 & 5830 & 4.0 & 1.0 & -0.06 & 0.05 & 34 & 0.05 & 8 & $\ldots$ & -0.03 & 0.01 & 4 & 0.04 & 0.05 & 3 \\
\hline HD 135694 & 5520 & 3.9 & 0.9 & -0.23 & 0.06 & 24 & 0.06 & 5 & $\begin{array}{l}\cdots \\
\ldots\end{array}$ & 0.07 & 0.01 & 3 & 0.19 & 0.09 & 2 \\
\hline HD 138750 & 6130 & 3.8 & 1.1 & -0.18 & 0.05 & 33 & 0.04 & 8 & $\ldots$ & -0.02 & 0.00 & 3 & 0.24 & 0.05 & 3 \\
\hline HD 140209 & 5710 & 4.0 & 1.1 & -0.14 & 0.05 & 28 & 0.03 & 5 & $\ldots$ & 0.01 & 0.05 & 5 & 0.23 & 0.08 & 4 \\
\hline HD 149105 & 5930 & 3.8 & 1.0 & -0.05 & 0.04 & 30 & 0.02 & 7 & 0.18 & -0.03 & 0.04 & 3 & -0.03 & 0.01 & 3 \\
\hline HD 149890 & 6030 & 4.0 & 1.1 & -0.20 & 0.05 & 33 & 0.03 & 6 & 0.17 & 0.00 & 0.04 & 3 & 0.07 & 0.07 & 3 \\
\hline HD 156617 & 5780 & 3.9 & 1.0 & -0.03 & 0.04 & 33 & 0.03 & 7 & 0.08 & -0.09 & 0.05 & 5 & 0.07 & 0.07 & 4 \\
\hline HD 156893 & 5300 & 3.8 & 0.9 & -0.21 & 0.04 & 36 & 0.03 & 8 & 0.22 & 0.02 & 0.05 & 4 & 0.23 & 0.07 & 4 \\
\hline HD 157214 & 5640 & 4.0 & 0.8 & -0.36 & 0.04 & 33 & 0.02 & 6 & 0.42 & 0.07 & 0.04 & 3 & 0.32 & 0.03 & 4 \\
\hline $\mathrm{BD}+403374$ & 5050 & 4.6 & 0.8 & -0.43 & 0.05 & 31 & 0.06 & 4 & $\ldots$ & 0.05 & 0.05 & 3 & 0.27 & 0.02 & 3 \\
\hline HD 171009 & 5840 & 4.0 & 1.0 & -0.35 & 0.04 & 32 & 0.04 & 6 & $\ldots$ & -0.01 & 0.04 & 3 & 0.19 & 0.04 & 3 \\
\hline HD 171242 & 5920 & 3.8 & 0.9 & -0.22 & 0.06 & 22 & 0.05 & 6 & 0.22 & 0.05 & 0.01 & 3 & 0.21 & 0.04 & 3 \\
\hline HD 178478 & 5550 & 3.6 & 1.0 & -0.50 & 0.03 & 24 & 0.04 & 5 & 0.40 & 0.11 & 0.02 & 3 & 0.35 & 0.04 & 4 \\
\hline HD 188326 & 5310 & 3.8 & 0.9 & -0.18 & 0.05 & 35 & 0.04 & 6 & 0.21 & 0.05 & 0.06 & 3 & 0.22 & 0.05 & 4 \\
\hline HD 206373 & 5900 & 3.8 & 1.1 & -0.16 & 0.04 & 26 & 0.02 & 5 & 0.15 & 0.06 & 0.03 & 3 & 0.09 & 0.08 & 4 \\
\hline HD 210483 & 5850 & 4.0 & 0.9 & -0.04 & 0.04 & 29 & 0.06 & 4 & $\ldots$ & -0.04 & 0.05 & 5 & 0.06 & 0.10 & 4 \\
\hline HD 211476 & 5840 & 4.2 & 0.9 & -0.10 & 0.05 & 32 & 0.03 & 7 & . & -0.03 & 0.02 & 3 & 0.01 & 0.03 & 4 \\
\hline HD 217511 & 6460 & 3.7 & 1.4 & -0.12 & 0.03 & 20 & 0.05 & 6 & 0.30 & 0.06 & 0.03 & 2 & & $\ldots$ & ... \\
\hline HD 219175 & 6050 & 4.2 & 0.9 & -0.24 & 0.05 & 30 & 0.03 & 5 & $\ldots$ & -0.04 & 0.04 & 5 & 0.05 & 0.04 & 4 \\
\hline \multicolumn{16}{|l|}{ Thin-disc stars } \\
\hline HD 115383 & 6090 & 4.0 & 1.2 & 0.20 & 0.05 & 29 & 0.05 & 8 & 0.02 & 0.00 & 0.01 & 4 & 0.08 & 0.07 & 3 \\
\hline HD 127334 & 5610 & 4.0 & 0.8 & 0.24 & 0.05 & 33 & 0.07 & 7 & -0.15 & 0.01 & 0.06 & 5 & 0.08 & 0.11 & 4 \\
\hline HD 136064 & 6090 & 3.9 & 1.1 & 0.05 & 0.06 & 30 & 0.04 & 7 & $\ldots$ & 0.02 & 0.03 & 3 & 0.16 & 0.14 & 2 \\
\hline HD 163989 & 6240 & 3.7 & 1.3 & 0.06 & 0.04 & 30 & 0.03 & 8 & 0.16 & 0.04 & 0.07 & 3 & 0.24 & 0.14 & 3 \\
\hline HD 187013 & 6290 & 3.6 & 1.3 & 0.00 & 0.05 & 22 & 0.04 & 7 & $\ldots$ & 0.01 & 0.04 & 3 & 0.27 & 0.01 & 2 \\
\hline HD 187691 & 6140 & 3.9 & 1.2 & 0.20 & 0.05 & 35 & 0.05 & 8 & 0.02 & 0.01 & 0.02 & 5 & 0.22 & 0.09 & 3 \\
\hline HD 200790 & 6190 & 3.9 & 1.3 & 0.08 & 0.04 & 32 & 0.02 & 8 & $\ldots$ & 0.05 & 0.05 & 3 & 0.32 & 0.09 & 2 \\
\hline HD 220117 & 6480 & 3.4 & 1.2 & 0.22 & 0.04 & 23 & 0.04 & 6 & $\ldots$ & $\ldots$ & $\ldots$ & $\ldots$ & $\ldots$ & $\ldots$ & ... \\
\hline \multicolumn{16}{|c|}{ Thick-disc stars } \\
\hline HD 150433 & 5650 & 4.2 & 0.9 & -0.34 & 0.05 & 37 & 0.05 & 7 & $\ldots$ & 0.07 & 0.01 & 3 & 0.31 & 0.04 & 4 \\
\hline HD 181047 & 5550 & 4.2 & 0.8 & 0.01 & 0.05 & 35 & 0.04 & 6 & $\ldots$ & -0.02 & 0.02 & 5 & 0.06 & 0.05 & 3 \\
\hline HD 186411 & 5860 & 3.7 & 1.2 & 0.11 & 0.05 & 34 & 0.03 & 8 & 0.00 & 0.00 & 0.01 & 5 & 0.16 & 0.05 & 3 \\
\hline HD 195019 & 5750 & 4.0 & 0.9 & 0.11 & 0.03 & 37 & 0.04 & 8 & 0.10 & -0.10 & 0.01 & 5 & 0.07 & 0.08 & 4 \\
\hline HD 198300 & 5830 & 4.1 & 0.9 & -0.44 & 0.04 & 38 & 0.04 & 8 & $\ldots$ & -0.03 & 0.03 & 3 & 0.11 & 0.05 & 4 \\
\hline
\end{tabular}


R. Ženovienè et al.: Stellar substructures in the solar neighbourhood. IV.

Table 4. continued.

\begin{tabular}{|c|c|c|c|c|c|c|c|c|c|c|c|c|c|c|c|}
\hline Star & {$[\mathrm{Al} / \mathrm{Fe}]$} & $\sigma$ & $n$ & {$[\mathrm{Si} / \mathrm{Fe}]$} & $\sigma$ & $n$ & {$[\mathrm{Ca} / \mathrm{Fe}]$} & $\sigma$ & $n$ & {$[\mathrm{Sc} / \mathrm{Fe}]$} & $\sigma$ & $n$ & {$[\mathrm{Tit} / \mathrm{Fe}]$} & $\sigma$ & $n$ \\
\hline \multicolumn{16}{|l|}{ Group 1 stars } \\
\hline HD 3795 & 0.38 & 0.03 & 3 & 0.20 & 0.05 & 16 & 0.25 & 0.05 & 7 & 0.16 & 0.04 & 10 & 0.28 & 0.05 & 15 \\
\hline HD 4607 & 0.03 & & 1 & 0.06 & 0.06 & 8 & 0.11 & 0.06 & 6 & 0.02 & 0.06 & 6 & 0.10 & 0.05 & 8 \\
\hline HD 15777 & 0.20 & 0.05 & 2 & 0.18 & 0.05 & 20 & 0.15 & 0.04 & 9 & 0.22 & 0.06 & 12 & 0.18 & 0.07 & 13 \\
\hline HD 22872 & -0.05 & 0.04 & 3 & 0.03 & 0.05 & 19 & -0.01 & 0.06 & 9 & 0.02 & 0.05 & 12 & -0.02 & 0.05 & 14 \\
\hline HD 25123 & 0.03 & 0.01 & 2 & 0.00 & 0.04 & 20 & 0.00 & 0.05 & 11 & 0.04 & 0.04 & 10 & 0.02 & 0.05 & 18 \\
\hline HD 40040 & 0.26 & 0.01 & 3 & 0.16 & 0.05 & 19 & 0.21 & 0.06 & 11 & 0.21 & 0.05 & 12 & 0.30 & 0.06 & 17 \\
\hline HD 49409 & 0.04 & 0.06 & 2 & 0.05 & 0.04 & 15 & 0.13 & 0.04 & 10 & 0.10 & 0.05 & 10 & 0.16 & 0.05 & 12 \\
\hline HD 52711 & -0.05 & 0.04 & 4 & 0.04 & 0.05 & 20 & 0.06 & 0.05 & 11 & -0.01 & 0.05 & 12 & 0.02 & 0.05 & 18 \\
\hline HD 60779 & 0.01 & 0.04 & 2 & 0.04 & 0.05 & 19 & 0.07 & 0.05 & 8 & 0.01 & 0.05 & 11 & 0.07 & 0.05 & 15 \\
\hline HD 67088 & -0.02 & 0.00 & 2 & 0.01 & 0.05 & 21 & 0.05 & 0.04 & 10 & 0.00 & 0.05 & 12 & -0.04 & 0.05 & 16 \\
\hline HD 67587 & 0.11 & 0.06 & 3 & 0.09 & 0.05 & 16 & 0.14 & 0.05 & 9 & 0.05 & 0.05 & 8 & 0.10 & 0.05 & 13 \\
\hline HD 76095 & 0.18 & 0.01 & 2 & 0.08 & 0.05 & 21 & 0.09 & 0.05 & 10 & 0.07 & 0.04 & 10 & 0.08 & 0.05 & 18 \\
\hline HD 77408 & 0.04 & 0.04 & 3 & 0.01 & 0.06 & 14 & 0.05 & 0.04 & 7 & 0.08 & 0.05 & 9 & 0.11 & 0.05 & 8 \\
\hline HD 78558 & 0.27 & 0.05 & 3 & 0.22 & 0.05 & 18 & 0.22 & 0.05 & 9 & 0.14 & 0.05 & 12 & 0.29 & 0.05 & 16 \\
\hline HD 88371 & 0.25 & 0.03 & 3 & 0.16 & 0.05 & 20 & 0.22 & 0.06 & 11 & 0.22 & 0.05 & 9 & 0.27 & 0.05 & 24 \\
\hline HD 88446 & 0.06 & 0.04 & 3 & 0.09 & 0.05 & 20 & 0.12 & 0.05 & 9 & 0.06 & 0.05 & 12 & 0.14 & 0.06 & 10 \\
\hline HD 90508 & 0.16 & 0.04 & 3 & 0.10 & 0.05 & 21 & 0.14 & 0.04 & 11 & 0.05 & 0.04 & 11 & 0.13 & 0.05 & 18 \\
\hline HD 109498 & 0.06 & 0.03 & 3 & 0.03 & 0.06 & 14 & 0.01 & 0.06 & 7 & 0.02 & 0.02 & 8 & 0.02 & 0.06 & 7 \\
\hline HD 111367 & 0.07 & 0.05 & 4 & 0.03 & 0.04 & 20 & 0.04 & 0.04 & 10 & 0.04 & 0.04 & 11 & 0.05 & 0.05 & 14 \\
\hline HD 135694 & 0.19 & 0.02 & 3 & 0.07 & 0.06 & 11 & 0.12 & 0.06 & 7 & 0.03 & 0.04 & 8 & 0.07 & 0.06 & 6 \\
\hline HD 138750 & -0.03 & 0.03 & 2 & 0.03 & 0.05 & 20 & 0.12 & 0.05 & 9 & 0.04 & 0.04 & 11 & 0.12 & 0.05 & 10 \\
\hline HD 140209 & 0.10 & $\ldots$ & 1 & 0.08 & 0.05 & 19 & 0.09 & 0.06 & 7 & 0.07 & 0.05 & 9 & 0.08 & 0.04 & 12 \\
\hline HD 149105 & -0.01 & 0.03 & 2 & -0.03 & 0.04 & 15 & 0.04 & 0.07 & 5 & 0.01 & 0.04 & 8 & -0.01 & 0.05 & 7 \\
\hline HD 149890 & 0.00 & 0.04 & 3 & 0.01 & 0.05 & 18 & 0.01 & 0.04 & 7 & -0.05 & 0.04 & 7 & -0.02 & 0.05 & 6 \\
\hline HD 156617 & 0.06 & 0.04 & 4 & -0.01 & 0.04 & 18 & 0.03 & 0.05 & 11 & -0.02 & 0.04 & 10 & 0.03 & 0.05 & 15 \\
\hline HD 156893 & 0.29 & 0.04 & 3 & 0.14 & 0.04 & 20 & 0.16 & 0.05 & 11 & 0.18 & 0.05 & 11 & 0.19 & 0.05 & 25 \\
\hline HD 157214 & 0.31 & 0.05 & 2 & 0.22 & 0.04 & 16 & 0.22 & 0.06 & 8 & 0.15 & 0.04 & 9 & 0.26 & 0.04 & 12 \\
\hline $\mathrm{BD}+403374$ & 0.38 & 0.03 & 3 & 0.20 & 0.05 & 18 & 0.23 & 0.04 & 8 & 0.24 & 0.04 & 8 & 0.38 & 0.07 & 22 \\
\hline HD 171009 & 0.12 & $\ldots$ & 1 & 0.12 & 0.04 & 15 & 0.15 & 0.06 & 7 & 0.13 & 0.04 & 7 & 0.19 & 0.03 & 8 \\
\hline HD 171242 & 0.01 & 0.04 & 2 & 0.10 & 0.05 & 11 & 0.14 & 0.04 & 7 & 0.02 & 0.05 & 7 & 0.11 & 0.04 & 10 \\
\hline HD 178478 & 0.33 & 0.04 & 3 & 0.20 & 0.05 & 14 & 0.25 & 0.05 & 7 & 0.06 & 0.06 & 7 & 0.31 & 0.06 & 8 \\
\hline HD 188326 & 0.22 & 0.00 & 2 & 0.13 & 0.03 & 15 & 0.12 & 0.05 & 7 & 0.07 & 0.05 & 6 & 0.20 & 0.05 & 13 \\
\hline HD 206373 & 0.02 & 0.03 & 4 & 0.04 & 0.05 & 15 & 0.05 & 0.02 & 6 & 0.04 & 0.04 & 8 & 0.05 & 0.03 & 7 \\
\hline HD 210483 & 0.07 & 0.03 & 3 & 0.00 & 0.05 & 17 & 0.05 & 0.06 & 7 & 0.02 & 0.04 & 8 & 0.04 & 0.05 & 18 \\
\hline HD 211476 & 0.05 & 0.01 & 2 & -0.01 & 0.04 & 16 & 0.02 & 0.03 & 6 & -0.03 & 0.04 & 8 & 0.01 & 0.03 & 9 \\
\hline HD 217511 & 0.00 & 0.04 & 2 & 0.03 & 0.05 & 17 & 0.06 & 0.05 & 9 & 0.10 & 0.05 & 7 & 0.02 & 0.05 & 6 \\
\hline HD 219175 & -0.06 & $\ldots$ & 1 & -0.01 & 0.06 & 15 & 0.01 & 0.05 & 7 & -0.02 & 0.03 & 9 & 0.02 & 0.04 & 6 \\
\hline \multicolumn{16}{|l|}{ Thin-disc stars } \\
\hline HD 115383 & & & & 0.06 & 0.05 & 13 & 0.08 & 0.04 & 8 & 0.00 & 0.06 & 9 & 0.07 & 0.04 & 8 \\
\hline HD 127334 & 0.07 & 0.03 & 2 & 0.03 & 0.05 & 20 & 0.00 & 0.05 & 11 & -0.02 & 0.05 & 10 & -0.03 & 0.05 & 16 \\
\hline HD 136064 & -0.04 & 0.03 & 2 & 0.00 & 0.05 & 15 & 0.09 & 0.05 & 6 & 0.10 & 0.04 & 8 & -0.02 & 0.05 & 10 \\
\hline HD 163989 & 0.04 & 0.07 & 4 & 0.06 & 0.05 & 15 & 0.04 & 0.03 & 4 & 0.06 & 0.05 & 9 & 0.10 & 0.06 & 8 \\
\hline HD 187013 & -0.04 & 0.02 & 2 & 0.06 & 0.06 & 11 & 0.09 & 0.04 & 4 & 0.01 & 0.06 & 7 & 0.09 & 0.06 & 5 \\
\hline HD 187691 & 0.00 & 0.06 & 3 & 0.03 & 0.05 & 18 & 0.05 & 0.02 & 8 & 0.01 & 0.05 & 12 & 0.03 & 0.05 & 12 \\
\hline HD 200790 & 0.01 & 0.01 & 2 & 0.04 & 0.05 & 16 & 0.01 & 0.04 & 7 & 0.05 & 0.05 & 9 & 0.00 & 0.04 & 7 \\
\hline HD 220117 & 0.01 & $\ldots$ & 1 & 0.00 & 0.05 & 9 & 0.10 & 0.05 & 5 & 0.06 & 0.04 & 4 & 0.12 & 0.05 & 6 \\
\hline \multicolumn{16}{|c|}{ Thick-disc stars } \\
\hline HD 150433 & 0.10 & $\ldots$ & 1 & 0.17 & 0.05 & 18 & 0.22 & 0.05 & 9 & 0.19 & 0.05 & 9 & 0.28 & 0.05 & 18 \\
\hline HD 181047 & 0.05 & 0.02 & 2 & 0.03 & 0.04 & 20 & 0.05 & 0.05 & 11 & -0.02 & 0.05 & 11 & -0.01 & 0.05 & 23 \\
\hline HD 186411 & 0.05 & 0.02 & 3 & 0.00 & 0.05 & 20 & 0.11 & 0.04 & 8 & 0.02 & 0.04 & 9 & 0.03 & 0.05 & 18 \\
\hline HD 195019 & -0.02 & 0.03 & 3 & -0.01 & 0.04 & 20 & 0.02 & 0.05 & 10 & -0.04 & 0.05 & 11 & -0.02 & 0.05 & 25 \\
\hline HD 198300 & 0.18 & 0.05 & 4 & 0.03 & 0.03 & 19 & 0.03 & 0.05 & 9 & -0.01 & 0.05 & 11 & 0.1 & 0.06 & 11 \\
\hline
\end{tabular}


Table 4. continued.

\begin{tabular}{|c|c|c|c|c|c|c|c|c|c|c|c|c|c|c|c|}
\hline Star & [TiII/Fe] & $\sigma$ & $n$ & {$[\mathrm{~V} / \mathrm{Fe}]$} & $\sigma$ & $n$ & {$[\mathrm{Cr} / \mathrm{Fe}]$} & $\sigma$ & $n$ & {$[\mathrm{Co} / \mathrm{Fe}]$} & $\sigma$ & $n$ & {$[\mathrm{Ni} / \mathrm{Fe}]$} & $\sigma$ & $n$ \\
\hline \multicolumn{16}{|l|}{ Group 1 stars } \\
\hline HD 3795 & 0.20 & 0.06 & 4 & 0.15 & 0.05 & 11 & 0.07 & 0.05 & 13 & 0.15 & 0.03 & 6 & 0.04 & 0.05 & 23 \\
\hline HD 4607 & 0.06 & 0.02 & 3 & 0.03 & 0.03 & 5 & -0.01 & 0.06 & 13 & -0.06 & 0.05 & 5 & -0.04 & 0.05 & 15 \\
\hline HD 15777 & 0.22 & 0.03 & 4 & 0.12 & 0.05 & 8 & 0.02 & 0.05 & 17 & 0.12 & 0.05 & 8 & 0.00 & 0.05 & 27 \\
\hline HD 22872 & -0.03 & 0.06 & 4 & 0.02 & 0.05 & 13 & 0.00 & 0.05 & 19 & -0.05 & 0.05 & 10 & -0.04 & 0.05 & 30 \\
\hline HD 25123 & 0.02 & 0.04 & 4 & 0.00 & 0.04 & 10 & 0.00 & 0.06 & 17 & 0.00 & 0.05 & 9 & -0.02 & 0.06 & 30 \\
\hline HD 40040 & 0.26 & 0.04 & 4 & 0.14 & 0.06 & 12 & 0.03 & 0.06 & 17 & 0.11 & 0.05 & 6 & 0.04 & 0.04 & 28 \\
\hline HD 49409 & 0.08 & 0.02 & 4 & 0.14 & 0.05 & 10 & 0.02 & 0.05 & 14 & 0.03 & 0.03 & 7 & -0.01 & 0.05 & 19 \\
\hline HD 52711 & 0.03 & 0.06 & 4 & -0.02 & 0.05 & 12 & 0.01 & 0.05 & 18 & -0.02 & 0.05 & 10 & -0.02 & 0.05 & 29 \\
\hline HD 60779 & 0.03 & 0.05 & 4 & 0.06 & 0.06 & 9 & 0.01 & 0.06 & 15 & -0.03 & 0.06 & 9 & -0.02 & 0.05 & 28 \\
\hline HD 67088 & -0.05 & 0.06 & 4 & -0.06 & 0.06 & 14 & 0.00 & 0.05 & 19 & -0.07 & 0.04 & 9 & -0.05 & 0.05 & 29 \\
\hline HD 67587 & 0.06 & 0.02 & 4 & 0.13 & 0.05 & 6 & 0.03 & 0.06 & 14 & 0.06 & 0.04 & 5 & -0.03 & 0.05 & 23 \\
\hline HD 76095 & 0.12 & 0.04 & 3 & 0.06 & 0.05 & 12 & 0.05 & 0.05 & 17 & 0.05 & 0.04 & 9 & -0.02 & 0.05 & 30 \\
\hline HD 77408 & 0.10 & 0.07 & 3 & 0.07 & 0.05 & 5 & -0.02 & 0.05 & 11 & 0.03 & 0.04 & 6 & -0.05 & 0.05 & 14 \\
\hline HD 78558 & 0.20 & 0.05 & 4 & 0.07 & 0.05 & 12 & 0.04 & 0.06 & 18 & 0.08 & 0.04 & 8 & 0.02 & 0.05 & 28 \\
\hline HD 88371 & 0.27 & 0.02 & 4 & 0.13 & 0.04 & 14 & 0.04 & 0.06 & 18 & 0.07 & 0.03 & 10 & 0.04 & 0.05 & 28 \\
\hline HD 88446 & 0.04 & 0.02 & 4 & 0.02 & 0.03 & 5 & 0.00 & 0.05 & 14 & 0.04 & 0.02 & 5 & -0.05 & 0.04 & 23 \\
\hline HD 90508 & 0.07 & 0.04 & 4 & 0.06 & 0.05 & 13 & 0.01 & 0.05 & 18 & 0.03 & 0.05 & 10 & -0.02 & 0.05 & 30 \\
\hline HD 109498 & 0.01 & 0.03 & 4 & 0.00 & 0.04 & 6 & 0.01 & 0.07 & 15 & 0.01 & 0.03 & 4 & -0.04 & 0.06 & 18 \\
\hline HD 111367 & -0.01 & 0.04 & 4 & 0.01 & 0.06 & 7 & -0.01 & 0.04 & 16 & -0.02 & 0.05 & 8 & -0.04 & 0.05 & 28 \\
\hline HD 135694 & 0.05 & 0.00 & 2 & -0.03 & 0.05 & 9 & 0.02 & 0.04 & 12 & 0.01 & 0.03 & 6 & -0.07 & 0.05 & 14 \\
\hline HD 138750 & 0.08 & 0.08 & 4 & 0.04 & 0.05 & 5 & 0.00 & 0.05 & 14 & -0.02 & 0.05 & 6 & -0.06 & 0.05 & 24 \\
\hline HD 140209 & 0.04 & 0.03 & 3 & 0.04 & 0.05 & 9 & 0.04 & 0.05 & 15 & 0.01 & 0.05 & 7 & -0.03 & 0.06 & 26 \\
\hline HD 149105 & -0.06 & 0.03 & 2 & -0.08 & 0.01 & 3 & -0.01 & 0.09 & 15 & -0.08 & 0.06 & 5 & -0.04 & 0.03 & 24 \\
\hline HD 149890 & -0.01 & 0.02 & 3 & -0.02 & 0.04 & 5 & -0.02 & 0.07 & 13 & -0.06 & 0.04 & 9 & -0.07 & 0.05 & 21 \\
\hline HD 156617 & -0.04 & 0.05 & 4 & -0.05 & 0.04 & 12 & 0.02 & 0.05 & 17 & -0.05 & 0.03 & 9 & -0.07 & 0.05 & 29 \\
\hline HD 156893 & 0.17 & 0.05 & 4 & 0.08 & 0.05 & 14 & 0.02 & 0.06 & 16 & 0.05 & 0.04 & 11 & 0.00 & 0.05 & 27 \\
\hline HD 157214 & 0.10 & 0.01 & 2 & 0.13 & 0.03 & 5 & 0.02 & 0.08 & 12 & 0.16 & 0.03 & 5 & 0.03 & 0.06 & 19 \\
\hline $\mathrm{BD}+403374$ & 0.30 & 0.04 & 3 & 0.24 & 0.04 & 7 & 0.10 & 0.05 & 13 & 0.19 & 0.04 & 8 & 0.04 & 0.06 & 24 \\
\hline HD 171009 & 0.11 & 0.04 & 3 & 0.07 & 0.04 & 4 & -0.02 & 0.08 & 13 & 0.06 & 0.08 & 5 & -0.03 & 0.05 & 16 \\
\hline HD 171242 & 0.03 & 0.05 & 4 & 0.03 & 0.04 & 8 & 0.03 & 0.04 & 12 & 0.05 & 0.05 & 4 & -0.09 & 0.05 & 15 \\
\hline HD 178478 & 0.21 & 0.01 & 3 & 0.16 & 0.05 & 8 & 0.10 & 0.06 & 9 & 0.07 & 0.03 & 4 & -0.03 & 0.05 & 18 \\
\hline HD 188326 & 0.27 & 0.03 & 4 & 0.14 & 0.06 & 9 & 0.05 & 0.05 & 15 & 0.10 & 0.04 & 7 & 0.02 & 0.05 & 21 \\
\hline HD 206373 & 0.03 & 0.05 & 4 & 0.01 & 0.05 & 5 & -0.04 & 0.05 & 13 & -0.03 & 0.04 & 4 & -0.04 & 0.04 & 18 \\
\hline HD 210483 & 0.01 & 0.04 & 4 & -0.06 & 0.02 & 7 & 0.01 & 0.07 & 14 & -0.04 & 0.05 & 9 & -0.06 & 0.05 & 26 \\
\hline HD 211476 & 0.00 & 0.04 & 4 & -0.03 & 0.04 & 6 & 0.01 & 0.05 & 16 & -0.04 & 0.04 & 7 & -0.04 & 0.04 & 22 \\
\hline HD 217511 & 0.06 & 0.01 & 2 & $\ldots$ & $\ldots$ & $\ldots$ & 0.00 & 0.05 & 8 & 0.04 & 0.01 & 4 & -0.01 & 0.05 & 13 \\
\hline HD 219175 & -0.03 & 0.03 & 3 & -0.02 & 0.05 & 8 & -0.05 & 0.05 & 9 & -0.04 & 0.03 & 3 & -0.08 & 0.05 & 22 \\
\hline \multicolumn{16}{|l|}{ Thin-disc stars } \\
\hline HD 115383 & & 0.03 & 3 & & 0.06 & 7 & 0.02 & 0.06 & 16 & 0.02 & 0.05 & 5 & -0.01 & 0.04 & 20 \\
\hline HD 127334 & -0.07 & 0.04 & 4 & -0.03 & 0.05 & 13 & 0.03 & 0.05 & 15 & 0.02 & 0.04 & 8 & 0.02 & 0.05 & 26 \\
\hline HD 136064 & 0.05 & 0.03 & 3 & 0.01 & 0.05 & 8 & 0.03 & 0.05 & 12 & -0.04 & 0.03 & 6 & -0.04 & 0.05 & 16 \\
\hline HD 163989 & 0.08 & 0.03 & 4 & 0.02 & 0.08 & 4 & -0.03 & 0.05 & 11 & -0.03 & 0.05 & 6 & -0.07 & 0.05 & 23 \\
\hline HD 187013 & 0.09 & 0.01 & 2 & $\ldots$ & $\ldots$ & $\ldots$ & 0.02 & 0.06 & 9 & $\ldots$ & $\ldots$ & $\ldots$ & -0.04 & 0.05 & 13 \\
\hline HD 187691 & 0.02 & 0.03 & 4 & -0.04 & 0.05 & 7 & 0.00 & 0.04 & 17 & -0.07 & 0.06 & 6 & -0.03 & 0.05 & 28 \\
\hline HD 200790 & -0.04 & 0.03 & 4 & -0.04 & 0.05 & 5 & 0.01 & 0.04 & 15 & -0.04 & 0.05 & 6 & -0.03 & 0.04 & 23 \\
\hline HD 220117 & $\ldots$ & $\ldots$ & $\ldots$ & $\ldots$ & $\ldots$ & $\ldots$ & 0.00 & 0.06 & 7 & $\ldots$ & $\ldots$ & $\ldots$ & -0.04 & 0.04 & 9 \\
\hline \multicolumn{16}{|c|}{ Thick-disc stars } \\
\hline HD 150433 & 0.26 & & 2 & 0.17 & 0.05 & 8 & 0.07 & 0.05 & 16 & & 0.05 & 7 & & 0.05 & 25 \\
\hline HD 181047 & -0.05 & 0.05 & 4 & -0.02 & 0.05 & 14 & 0.05 & 0.06 & 18 & -0.02 & 0.06 & 11 & -0.01 & 0.05 & 26 \\
\hline HD 186411 & 0.02 & 0.03 & 4 & -0.01 & 0.06 & 12 & 0.01 & 0.06 & 17 & -0.04 & 0.05 & 7 & -0.07 & 0.05 & 27 \\
\hline HD 195019 & -0.05 & 0.05 & 4 & -0.05 & 0.05 & 12 & 0.01 & 0.05 & 19 & -0.05 & 0.05 & 8 & -0.06 & 0.05 & 30 \\
\hline HD 198300 & 0.03 & 0.05 & 4 & 0.01 & 0.04 & 7 & -0.04 & 0.06 & 16 & 0.04 & 0.02 & 6 & -0.06 & 0.05 & 26 \\
\hline
\end{tabular}


R. Ženovienè et al.: Stellar substructures in the solar neighbourhood. IV.

Table 5. Elemental abundances of neutron-capture elements for programme and comparison stars.

\begin{tabular}{|c|c|c|c|c|c|c|c|c|c|c|c|c|c|}
\hline Star & {$[\mathrm{Y} / \mathrm{Fe}]$} & $\sigma$ & $n$ & {$[\mathrm{ZrI} / \mathrm{Fe}]$} & $\sigma$ & $n$ & {$[\mathrm{ZrII} / \mathrm{Fe}]$} & $\sigma$ & $n$ & {$[\mathrm{Ba} / \mathrm{Fe}]$} & {$[\mathrm{La} / \mathrm{Fe}]$} & $\sigma$ & $n$ \\
\hline \multicolumn{14}{|l|}{ Group 1 stars } \\
\hline HD 3795 & -0.03 & 0.03 & 6 & 0.17 & 0.07 & 4 & 0.08 & 0.02 & 2 & -0.06 & 0.20 & 0.05 & 3 \\
\hline HD 4607 & -0.02 & 0.02 & 5 & 0.07 & 0.02 & 3 & 0.07 & 0.01 & 2 & 0.28 & -0.05 & 0.07 & 2 \\
\hline HD 15777 & -0.13 & 0.06 & 4 & 0.13 & 0.00 & 3 & 0.10 & 0.09 & 2 & -0.14 & -0.01 & 0.01 & 3 \\
\hline HD 22872 & -0.12 & 0.01 & 6 & -0.06 & 0.08 & 4 & -0.01 & 0.09 & 2 & 0.00 & -0.16 & 0.05 & 3 \\
\hline HD 25123 & -0.07 & 0.05 & 7 & 0.07 & 0.02 & 2 & -0.04 & 0.01 & 2 & 0.06 & 0.00 & 0.04 & 4 \\
\hline HD 40040 & -0.22 & 0.03 & 4 & 0.03 & 0.01 & 2 & -0.05 & 0.05 & 2 & -0.13 & 0.02 & 0.06 & 2 \\
\hline HD 49409 & -0.21 & 0.08 & 2 & 0.19 & 0.05 & 2 & 0.11 & 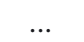 & 1 & 0.07 & -0.13 & & 1 \\
\hline HD 52711 & -0.16 & 0.03 & 7 & 0.08 & 0.05 & 5 & 0.05 & 0.04 & 2 & -0.08 & -0.06 & 0.06 & 4 \\
\hline HD 60779 & -0.06 & 0.06 & 7 & 0.05 & 0.03 & 3 & 0.01 & 0.04 & 2 & 0.11 & -0.04 & 0.06 & 4 \\
\hline HD 67088 & -0.20 & 0.04 & 7 & -0.20 & 0.03 & 3 & -0.15 & 0.04 & 2 & 0.00 & -0.03 & 0.05 & 4 \\
\hline HD 67587 & -0.16 & 0.05 & 4 & -0.10 & $\ldots$ & 1 & -0.18 & $\ldots$ & 1 & 0.08 & 0.01 & 0.00 & 3 \\
\hline HD 76095 & -0.05 & 0.06 & 5 & 0.00 & 0.05 & 3 & 0.00 & 0.04 & 2 & 0.24 & 0.14 & 0.01 & 2 \\
\hline HD 77408 & -0.08 & 0.01 & 3 & & & $\ldots$ & 0.17 & $\ldots$ & 1 & 0.21 & 0.07 & & 1 \\
\hline HD 78558 & -0.13 & 0.05 & 6 & 0.07 & 0.09 & 2 & -0.01 & 0.01 & 2 & -0.13 & -0.16 & 0.04 & 2 \\
\hline HD 88371 & 0.03 & 0.07 & 4 & 0.06 & 0.03 & 4 & 0.06 & 0.02 & 2 & -0.18 & -0.09 & $\ldots$ & 1 \\
\hline HD 88446 & 0.57 & 0.05 & 5 & 0.57 & & 1 & 0.56 & 0.01 & 2 & 1.04 & 0.70 & 0.08 & 4 \\
\hline HD 90508 & -0.19 & 0.05 & 6 & -0.06 & 0.05 & 4 & -0.01 & 0.01 & 2 & -0.05 & 0.02 & 0.04 & 3 \\
\hline HD 109498 & -0.09 & 0.02 & 6 & -0.02 & 0.06 & 3 & -0.03 & 0.04 & 2 & -0.07 & -0.01 & 0.07 & 2 \\
\hline HD 111367 & -0.17 & 0.05 & 6 & 0.00 & 0.06 & 3 & -0.02 & 0.03 & 2 & 0.06 & 0.06 & 0.05 & 2 \\
\hline HD 135694 & -0.20 & 0.07 & 3 & -0.01 & 0.07 & 4 & 0.01 & $\ldots$ & 1 & -0.04 & $\ldots$ & $\ldots$ & $\ldots$ \\
\hline HD 138750 & 0.00 & 0.04 & 7 & 0.16 & 0.03 & 3 & 0.04 & 0.07 & 2 & 0.19 & 0.04 & 0.05 & 4 \\
\hline HD 140209 & -0.13 & 0.03 & 4 & 0.00 & 0.04 & 3 & -0.14 & 0.06 & 2 & -0.24 & -0.07 & 0.02 & 3 \\
\hline HD 149105 & -0.16 & 0.04 & 5 & -0.02 & $\ldots$ & 1 & -0.03 & 0.01 & 2 & 0.11 & -0.07 & 0.00 & 2 \\
\hline HD 149890 & -0.15 & 0.04 & 6 & 0.09 & $\ldots$ & 1 & 0.07 & $\ldots$ & 1 & 0.00 & -0.02 & 0.01 & 2 \\
\hline HD 156617 & -0.21 & 0.05 & 6 & -0.09 & 0.04 & 2 & -0.07 & $\ldots$ & 1 & 0.03 & -0.07 & 0.08 & 3 \\
\hline HD 156893 & -0.03 & 0.06 & 6 & -0.02 & 0.04 & 6 & 0.04 & 0.02 & 2 & 0.21 & 0.15 & 0.07 & 3 \\
\hline HD 157214 & -0.13 & 0.07 & 5 & 0.08 & 0.06 & 4 & 0.05 & 0.03 & 2 & -0.26 & -0.06 & 0.06 & 2 \\
\hline $\mathrm{BD}+403374$ & -0.14 & 0.07 & 3 & 0.30 & 0.04 & 5 & 0.34 & $\ldots$ & 1 & $\ldots$ & 0.04 & $\ldots$ & 1 \\
\hline HD 171009 & -0.12 & 0.04 & 4 & 0.17 & 0.03 & 3 & 0.09 & $\ldots$ & 1 & 0.02 & 0.05 & 0.05 & 3 \\
\hline HD 171242 & -0.14 & 0.00 & 2 & 0.24 & $\ldots$ & 1 & 0.21 & 0.06 & 2 & $\ldots$ & 0.20 & 0.01 & 2 \\
\hline HD 178478 & -0.15 & 0.10 & 3 & 0.18 & 0.04 & 2 & $\ldots$ & $\ldots$ & $\ldots$ & -0.24 & 0.11 & 0.05 & 3 \\
\hline HD 188326 & -0.05 & 0.06 & 5 & 0.06 & 0.03 & 6 & 0.02 & $\ldots$ & 1 & 0.00 & 0.13 & 0.05 & 3 \\
\hline HD 206373 & -0.09 & 0.06 & 6 & 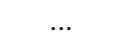 & 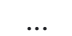 & $\ldots$ & -0.14 & $\ldots$ & 1 & 0.16 & -0.07 & 0.05 & 3 \\
\hline HD 210483 & -0.17 & 0.05 & 4 & 0.00 & 0.02 & 2 & -0.05 & 0.02 & 2 & -0.11 & -0.03 & 0.06 & 3 \\
\hline HD 211476 & -0.16 & 0.06 & 7 & -0.04 & 0.04 & 5 & 0.00 & 0.06 & 2 & -0.10 & 0.06 & 0.05 & 2 \\
\hline HD 217511 & 0.11 & 0.02 & 5 & $\ldots$ & $\ldots$ & $\ldots$ & $\ldots$ & $\ldots$ & $\ldots$ & $\ldots$ & 0.21 & 0.01 & 2 \\
\hline HD 219175 & -0.15 & 0.04 & 5 & -0.09 & $\ldots$ & 1 & -0.09 & 0.00 & 2 & 0.00 & 0.12 & 0.00 & 2 \\
\hline \multicolumn{14}{|l|}{ Thin-disc stars } \\
\hline HD 115383 & -0.17 & 0.06 & 5 & 0.00 & $\ldots$ & 1 & -0.03 & 0.05 & 2 & -0.05 & -0.09 & & 1 \\
\hline HD 127334 & -0.20 & 0.05 & 6 & -0.15 & 0.05 & 7 & -0.15 & 0.03 & 2 & $\ldots$ & -0.17 & 0.06 & 3 \\
\hline HD 136064 & 0.05 & 0.00 & 2 & $\ldots$ & $\ldots$ & $\ldots$ & 0.19 & 0.04 & 2 & 0.05 & 0.08 & $\ldots$ & 1 \\
\hline HD 163989 & 0.01 & 0.05 & 5 & $\ldots$ & $\ldots$ & $\ldots$ & 0.03 & 0.01 & 2 & 0.03 & 0.08 & 0.09 & 3 \\
\hline HD 187013 & -0.15 & 0.03 & 5 & -0.12 & $\ldots$ & 1 & -0.12 & $\ldots$ & 1 & 0.00 & 0.07 & 0.04 & 2 \\
\hline HD 187691 & -0.13 & 0.07 & 6 & -0.10 & $\ldots$ & 1 & -0.09 & 0.01 & 2 & -0.14 & -0.05 & 0.02 & 3 \\
\hline HD 200790 & -0.18 & 0.04 & 5 & -0.12 & $\ldots$ & 1 & -0.11 & 0.07 & 2 & -0.17 & -0.07 & 0.05 & 4 \\
\hline HD 220117 & $\ldots$ & $\ldots$ & ... & $\ldots$ & $\ldots$ & $\ldots$ & $\ldots$ & $\ldots$ & ... & -0.17 & $\ldots$ & $\ldots$ & ... \\
\hline \multicolumn{14}{|c|}{ Thick-disc stars } \\
\hline HD 150433 & -0.06 & 0.04 & 5 & 0.12 & 0.06 & 4 & 0.12 & $\ldots$ & 1 & -0.16 & 0.00 & 0.04 & 2 \\
\hline HD 181047 & 0.00 & 0.06 & 7 & -0.08 & 0.05 & 4 & -0.05 & 0.01 & 2 & -0.01 & -0.06 & 0.02 & 3 \\
\hline HD 186411 & -0.05 & 0.05 & 6 & -0.03 & 0.04 & 3 & -0.03 & 0.04 & 2 & 0.04 & 0.03 & 0.06 & 4 \\
\hline HD 195019 & -0.17 & 0.06 & 7 & -0.20 & 0.04 & 4 & -0.17 & 0.04 & 2 & -0.01 & -0.09 & 0.06 & 4 \\
\hline HD 198300 & -0.14 & 0.06 & 5 & -0.02 & 0.09 & 2 & $\ldots$ & $\ldots$ & $\ldots$ & 0.05 & -0.01 & 0.07 & 3 \\
\hline
\end{tabular}


Table 5. continued.

\begin{tabular}{|c|c|c|c|c|c|c|c|c|c|c|c|c|c|c|c|}
\hline Star & {$[\mathrm{Ce} / \mathrm{Fe}]$} & $\sigma$ & $n$ & {$[\mathrm{Pr} / \mathrm{Fe}]$} & $\sigma$ & $n$ & {$[\mathrm{Nd} / \mathrm{Fe}]$} & $\sigma$ & $n$ & {$[\mathrm{Sm} / \mathrm{Fe}]$} & $\sigma$ & $n$ & {$[\mathrm{Eu} / \mathrm{Fe}]$} & $\sigma$ & $n$ \\
\hline \multicolumn{16}{|l|}{ Group 1 stars } \\
\hline HD 3795 & 0.17 & 0.06 & 2 & 0.44 & 0.14 & 2 & 0.32 & 0.03 & 6 & 0.44 & 0.13 & 3 & 0.64 & 0.03 & 2 \\
\hline HD 4607 & -0.02 & 0.00 & 2 & 0.14 & & 1 & 0.08 & 0.04 & 4 & 0.11 & 0.01 & 2 & 0.03 & 0.04 & 2 \\
\hline HD 15777 & 0.04 & 0.02 & 3 & 0.14 & 0.05 & 2 & 0.12 & 0.05 & 5 & 0.18 & 0.05 & 3 & 0.30 & 0.04 & 2 \\
\hline HD 22872 & -0.09 & 0.09 & 3 & 0.02 & 0.06 & 2 & -0.10 & 0.02 & 7 & -0.01 & 0.08 & 3 & -0.07 & 0.06 & 2 \\
\hline HD 25123 & -0.08 & 0.05 & 5 & -0.05 & 0.01 & 2 & -0.06 & 0.05 & 5 & -0.06 & 0.04 & 3 & -0.04 & 0.00 & 2 \\
\hline HD 40040 & -0.05 & 0.06 & 3 & 0.24 & 0.02 & 2 & 0.09 & 0.05 & 5 & 0.21 & 0.05 & 2 & 0.28 & 0.03 & 2 \\
\hline HD 49409 & -0.04 & 0.06 & 2 & 0.23 & $\ldots$ & 1 & 0.25 & 0.03 & 3 & 0.24 & 0.07 & 3 & 0.33 & . & 1 \\
\hline HD 52711 & -0.09 & 0.05 & 4 & -0.01 & 0.12 & 2 & -0.04 & 0.05 & 5 & -0.01 & 0.03 & 3 & -0.08 & 0.04 & 2 \\
\hline HD 60779 & 0.03 & 0.04 & 3 & 0.19 & $\ldots$ & 1 & -0.02 & 0.07 & 6 & 0.01 & 0.03 & 3 & 0.01 & 0.02 & 2 \\
\hline HD 67088 & -0.10 & 0.03 & 4 & 0.06 & 0.03 & 2 & -0.08 & 0.05 & 6 & -0.03 & 0.06 & 3 & -0.01 & 0.08 & 2 \\
\hline HD 67587 & -0.06 & 0.05 & 2 & $\ldots$ & $\ldots$ & $\ldots$ & 0.07 & 0.07 & 3 & $\ldots$ & $\ldots$ & $\ldots$ & 0.02 & 0.06 & 2 \\
\hline HD 76095 & 0.13 & 0.04 & 4 & 0.16 & 0.06 & 2 & 0.16 & 0.07 & 5 & 0.15 & 0.01 & 2 & 0.22 & 0.04 & 2 \\
\hline HD 77408 & 0.06 & & 1 & 0.21 & & 1 & 0.20 & 0.02 & 3 & 0.22 & & 1 & 0.16 & 0.02 & 2 \\
\hline HD 78558 & -0.02 & 0.05 & 5 & 0.22 & 0.03 & 2 & 0.03 & 0.05 & 5 & 0.16 & 0.01 & 2 & 0.20 & 0.01 & 2 \\
\hline HD 88371 & 0.00 & 0.04 & 3 & 0.11 & $\ldots$ & 1 & 0.08 & 0.03 & 5 & 0.21 & 0.01 & 3 & 0.24 & $\ldots$ & 1 \\
\hline HD 88446 & 0.65 & 0.03 & 4 & 0.56 & 0.06 & 2 & 0.66 & 0.03 & 7 & 0.55 & 0.06 & 2 & 0.34 & 0.09 & 2 \\
\hline HD 90508 & -0.08 & 0.09 & 3 & 0.11 & 0.07 & 2 & 0.06 & 0.04 & 8 & 0.18 & 0.03 & 3 & 0.17 & 0.01 & 2 \\
\hline HD 109498 & 0.08 & 0.03 & 4 & 0.17 & 0.04 & 2 & 0.08 & 0.06 & 4 & 0.15 & 0.00 & 2 & 0.16 & 0.05 & 2 \\
\hline HD 111367 & 0.09 & $\ldots$ & 1 & 0.14 & 0.00 & 2 & -0.04 & 0.07 & 4 & 0.06 & 0.00 & 2 & 0.02 & 0.06 & 2 \\
\hline HD 135694 & 0.01 & 0.08 & 3 & 0.26 & & 1 & 0.00 & 0.05 & 4 & 0.21 & 0.08 & 3 & 0.23 & 0.11 & 2 \\
\hline HD 138750 & 0.03 & 0.08 & 3 & 0.12 & 0.07 & 2 & 0.04 & 0.04 & 5 & 0.04 & 0.05 & 3 & 0.01 & 0.01 & 2 \\
\hline HD 140209 & -0.04 & 0.03 & 5 & 0.08 & $\ldots$ & 1 & 0.00 & 0.03 & 5 & 0.05 & 0.04 & 3 & 0.09 & 0.04 & 2 \\
\hline HD 149105 & -0.09 & 0.02 & 2 & -0.1 & $\ldots$ & 1 & 0.02 & 0.03 & 4 & 0.10 & 0.11 & 2 & 0.08 & 0.05 & 2 \\
\hline HD 149890 & -0.03 & $\ldots$ & 1 & -0.02 & $\ldots$ & 1 & -0.02 & 0.02 & 4 & 0.07 & $\ldots$ & 1 & 0.02 & 0.00 & 2 \\
\hline HD 156617 & -0.07 & 0.02 & 4 & -0.13 & 0.04 & 2 & -0.06 & 0.04 & 5 & -0.01 & 0.02 & 3 & -0.02 & 0.05 & 2 \\
\hline HD 156893 & 0.04 & 0.09 & 3 & 0.26 & 0.08 & 2 & 0.20 & 0.02 & 8 & 0.34 & 0.01 & 2 & 0.38 & 0.02 & 2 \\
\hline HD 157214 & -0.08 & 0.03 & 3 & 0.13 & 0.01 & 2 & 0.04 & 0.06 & 5 & 0.27 & 0.05 & 3 & 0.28 & 0.02 & 2 \\
\hline $\mathrm{BD}+403374$ & 0.19 & 0.05 & 3 & $\ldots$ & $\ldots$ & $\ldots$ & 0.30 & 0.06 & 3 & 0.29 & 0.05 & 3 & 0.51 & 0.01 & 2 \\
\hline HD 171009 & 0.05 & 0.09 & 3 & 0.27 & $\ldots$ & 1 & 0.12 & 0.06 & 6 & 0.25 & 0.06 & 2 & 0.29 & 0.03 & 2 \\
\hline HD 171242 & 0.20 & 0.07 & 2 & $\ldots$ & $\ldots$ & $\ldots$ & 0.14 & 0.04 & 5 & 0.36 & $\ldots$ & 1 & 0.24 & $\ldots$ & 1 \\
\hline HD 178478 & -0.04 & 0.05 & 2 & $\ldots$ & $\ldots$ & $\ldots$ & -0.04 & 0.04 & 4 & $\ldots$ & $\ldots$ & $\ldots$ & 0.19 & 0.07 & 2 \\
\hline HD 188326 & 0.09 & 0.05 & 3 & 0.19 & 0.01 & 2 & 0.13 & 0.04 & 7 & 0.19 & 0.05 & 3 & 0.21 & 0.04 & 2 \\
\hline HD 206373 & -0.02 & 0.05 & 3 & 0.2 & 0.00 & 2 & -0.03 & 0.05 & 3 & -0.02 & 0.03 & 2 & -0.01 & $\ldots$ & 1 \\
\hline HD 210483 & -0.10 & 0.09 & 4 & 0 & $\ldots$ & 1 & -0.03 & 0.04 & 6 & -0.04 & $\ldots$ & 1 & -0.02 & $\ldots$ & 1 \\
\hline HD 211476 & -0.10 & 0.06 & 4 & 0.11 & $\ldots$ & 1 & 0.02 & 0.05 & 5 & 0.09 & 0.07 & 3 & 0.05 & 0.02 & 2 \\
\hline HD 217511 & 0.14 & $\ldots$ & 1 & $\ldots$ & $\ldots$ & $\ldots$ & 0.20 & $\ldots$ & 1 & 0.16 & $\ldots$ & 1 & 0.14 & 0.01 & 2 \\
\hline HD 219175 & -0.04 & 0.06 & 2 & 0.14 & $\ldots$ & 1 & -0.03 & 0.06 & 4 & 0.04 & 0.00 & 2 & 0.05 & 0.01 & 2 \\
\hline \multicolumn{16}{|l|}{ Thin-disc stars } \\
\hline HD 115383 & -0.19 & 0.01 & 2 & 0.03 & $\ldots$ & 1 & -0.13 & 0.03 & 4 & -0.15 & 0.03 & 3 & -0.09 & 0.01 & 2 \\
\hline HD 127334 & -0.20 & 0.03 & 4 & -0.14 & $\ldots$ & 1 & -0.17 & 0.05 & 6 & -0.21 & 0.04 & 2 & -0.19 & 0.06 & 2 \\
\hline HD 136064 & & & $\ldots$ & 0.10 & $\ldots$ & 1 & 0.05 & 0.07 & 4 & & $\ldots$ & $\ldots$ & 0.09 & 0.06 & 2 \\
\hline HD 163989 & 0.08 & 0.03 & 3 & 0.03 & $\ldots$ & 1 & 0.04 & 0.03 & 3 & 0.06 & 0.05 & 2 & -0.01 & 0.04 & 2 \\
\hline HD 187013 & -0.04 & $\ldots$ & 1 & -0.05 & 0.01 & 2 & -0.17 & 0.03 & 2 & -0.08 & 0.08 & 2 & -0.11 & 0.03 & 2 \\
\hline HD 187691 & -0.16 & 0.05 & 3 & -0.10 & $\ldots$ & 2 & -0.22 & 0.04 & 4 & -0.05 & $\ldots$ & 1 & -0.07 & 0.00 & 2 \\
\hline HD 200790 & -0.09 & 0.08 & 4 & -0.19 & 0.03 & 2 & -0.16 & 0.05 & 5 & -0.09 & 0.02 & 2 & -0.15 & 0.00 & 2 \\
\hline HD 220117 & $\ldots$ & $\cdots$ & $\cdots$ & $\cdots$ & $\cdots$ & $\cdots$ & $\cdots$ & $\cdots$ & ... & $\cdots$ & $\cdots$ & $\cdots$ & $\cdots$ & $\cdots$ & $\cdots$ \\
\hline \multicolumn{16}{|l|}{ Thick-disc stars } \\
\hline HD 150433 & -0.08 & & 1 & 0.37 & $\cdots$ & 1 & 0.12 & 0.03 & 4 & 0.34 & & 1 & 0.25 & 0.03 & 2 \\
\hline HD 181047 & -0.02 & 0.05 & 3 & 0.07 & $\ldots$ & 1 & -0.02 & 0.04 & 6 & 0.07 & 0.06 & 2 & 0.01 & 0.08 & 2 \\
\hline HD 186411 & -0.11 & 0.05 & 4 & -0.06 & 0.13 & 2 & -0.05 & 0.04 & 6 & -0.06 & 0.02 & 2 & -0.03 & 0.01 & 2 \\
\hline HD 195019 & -0.10 & 0.02 & 4 & -0.01 & 0.06 & 2 & -0.06 & 0.04 & 8 & -0.01 & 0.08 & 3 & -0.03 & 0.08 & 2 \\
\hline HD 198300 & -0.03 & 0.07 & 4 & 0.28 & 0.06 & 2 & 0.07 & 0.02 & 3 & 0.25 & 0.01 & 2 & 0.12 & 0.00 & 2 \\
\hline
\end{tabular}

